\title{
Array Processing for Radar: Achievements and Challenges
}

\author{
Ulrich Nickel \\ Fraunhofer Institute for Communication, Information Processing and Ergonomics (FKIE), Fraunhoferstrasse 20, \\ 53343 Wachtberg, Germany \\ Correspondence should be addressed to Ulrich Nickel; ulrich.nickel@fkie.fraunhofer.de
}

Received 26 March 2013; Accepted 26 July 2013

Academic Editor: Hang Hu

Copyright ( 2013 Ulrich Nickel. This is an open access article distributed under the Creative Commons Attribution License, which permits unrestricted use, distribution, and reproduction in any medium, provided the original work is properly cited.

Array processing for radar is well established in the literature, but only few of these algorithms have been implemented in real systems. The reason may be that the impact of these algorithms on the overall system must be well understood. For a successful implementation of array processing methods exploiting the full potential, the desired radar task has to be considered and all processing necessary for this task has to be eventually adapted. In this tutorial paper, we point out several viewpoints which are relevant in this context: the restrictions and the potential provided by different array configurations, the predictability of the transmission function of the array, the constraints for adaptive beamforming, the inclusion of monopulse, detection and tracking into the adaptive beamforming concept, and the assessment of superresolution methods with respect to their application in a radar system. The problems and achieved results are illustrated by examples from previous publications.

\section{Introduction}

Array processing is well established for radar. Publications of this topic have appeared for decades, and one might question what kind of advances we may still expect now. On the other hand, if we look at existing radar systems we will find very few methods implemented from the many ideas discussed in the literature. The reason may be that all processing elements of a radar system are linked, and it is not very useful to simply implement an isolated algorithm. The performance and the property of any algorithm will have an influence on the subsequent processing steps and on the radar operational modes. Predictability of the system performance with the new algorithms is a key issue for the radar designer. Advanced array processing for radar will therefore require to take these interrelationships into account and to adapt the related processing in order to achieve the maximum possible improvement. The standard handbooks on radar $[1,2]$ do not mention this problem. The book of Wirth [3] is an exception and mentions a number of the array processing techniques described below.

In this tutorial paper, viewpoints are presented which are relevant for the implementation of array processing methods. We do not present any new sophisticated algorithms, but for the established algorithms we give examples of the relations between array processing and preceding and subsequent radar processing. We point out the problems that have to be encountered and the solutions that need to be developed. We start with spatial sampling, that is, the antenna array that has to be designed to fulfill all requirements of the radar system. A modern radar is typically a multitasking system. So, the design of the array antenna has to fulfill multiple purposes in a compromise. In Section 3, we briefly review the approaches for deterministic pattern shaping which is the standard approach of antenna-based interference mitigation. It has the advantage of requiring little knowledge about the interference scenario, but very precise knowledge about the array transfer function ("the array manifold"). Adaptive beamforming $(\mathrm{ABF})$ is presented in Section 4. This approach requires little knowledge about the array manifold but needs to estimate the interference scenario from some training data. Superresolution for best resolution of multiple targets is sometimes also subsumed under adaptive beamforming as it resolves everything, interference and targets. These methods are considered in Section 5. We consider superresolution methods here solely for the purpose of improved parameter estimation. In Section 6, we briefly mention the canonical extension of $\mathrm{ABF}$ and superresolution to space-time array processing. Section 7 is the final and most important contribution. Here 
we point out how direction estimation must be modified if adaptive beams are used, and how the radar detector, the tracking algorithm, and the track management should be adapted for $\mathrm{ABF}$.

\section{Design Factors for Arrays}

Array processing starts with the array antenna. This is hardware and is a selected construction that cannot be altered. It must therefore be carefully designed to fulfill all requirements. Digital array processing requires digital array outputs. The number and quality of these receivers (e.g., linearity and number of ADC bits) determine the quality and the cost of the whole system. It may be desirable to design a fully digital array with $\mathrm{AD}$-converters at each antenna element. However, weight and cost will often lead to a system with reduced number of digital receivers. On the other hand, because of the $1 / R^{4}$-decay of the received power, radar needs antennas with high gain and high directional discrimination, which means arrays with many elements. There are different solutions to solve this contradiction.

(i) Thinned arrays: the angular discrimination of an array with a number of elements can be improved by increasing the separation of the elements and thus increasing the aperture of the antenna. Note that the thinned array has the same gain as the corresponding fully filled array.

(ii) Subarrays: element outputs are summed up in an analog manner into subarrays which are then ADconverted and processed digitally. The size and the shape of the subarrays are an important design criterion. The notion of an array with subarrays is very general and includes the case of steerable directional array elements.

2.1. Impact of the Dimensionality of the Array. Antenna elements may be arranged on a line (1-dimensional array), on a plane (a ring or a 2-dimensional planar array), on a curved surface (conformal array), or within a volume (3D-array, also called Crow's Nest antenna, [3, Section 4.6.1]). A 1dimensional array can only measure one independent angle; $2 \mathrm{D}$ and $3 \mathrm{D}$ arrays can measure the full polar coordinates in $\mathbb{R}^{3}$.

Antenna element design and the need for fixing elements mechanically lead to element patterns which are never omnidirectional. The elements have to be designed with patterns that allow a unique identification of the direction. Typically, a planar array can only observe a hemispherical half space. To achieve full spherical coverage, several planar arrays can be combined (multifacetted array), or a conformal or volume array may be used.

2.1.1. Arrays with Equal Patterns. For linear, planar, and volume arrays, elements with nearly equal patterns can be realized. These have the advantage that the knowledge of the element pattern is for many array processing methods not necessary. An equal complex value can be interpreted as a modified target complex amplitude, which is often a nuisance parameter. More important is that, if the element patterns are really absolutely equal, any cross-polar components of the signal are in all channels equal and fulfill the array model in the same way as the copolar components; that is, they produce no error effect.

2.1.2. Arrays with Unequal Array Patterns. This occurs typically by tilting the antenna elements as is done for conformal arrays. For a planar array, this tilt may be used to realize an array with polarization diversity. Single polarized elements are then mounted with orthogonal alignment at different positions. Such an array can provide some degree of dual polarization reception with single channel receivers (contrary to more costly fully polarimetric arrays with receivers for both polarizations for each channel).

Common to arrays with unequal patterns is that we have to know the element patterns for applying array processing methods. The full element pattern function is also called the array manifold. In particular, the cross-polar (or short $x$-pol) component has a different influence for each element. This means that if this component is not known and if the $x$-pol component is not sufficiently attenuated, it can be a significant source of error.

2.2. Thinned Arrays. To save the cost of receiving modules, sparse arrays are considered, that is, with fewer elements than the full populated $\lambda / 2$ grid. Because such a "thinned array" spans the same aperture, it has the same beamwidth. Hence, the angular accuracy and resolution are the same as the fully filled array. Due to the gaps, ambiguities or at least high sidelobes may arise. In early publications like [1], it was advocated to simply take out elements of the fully filled array. It was early recognized that this kind of thinning does not imply "sufficiently random" positions. Random positions on a $\lambda / 16$ grid as used in [3, Chapter 17] can provide quite acceptable patterns. Note that the array gain of a thinned array with $N$ elements is always $N$, and the average sidelobe level is $1 / N$. Today, we know from the theory of compressed sensing that a selection of sufficiently random positions can produce a unique reconstruction of a not too large number of impinging wave fields with high probability [5].

2.3. Arrays with Subarrays. If a high antenna gain with low sidelobes is desired one has to go back to the fully filled array. For large arrays with thousands of elements, the large number of digital channel constitutes a significant cost factor and a challenge for the resulting data rate. Therefore, often subarrays are formed, and all digital (adaptive) beamforming and sophisticated array processing methods are applied to the subarray outputs. Subarraying is a very general concept. At the elements, we may have phase shifters such that all subarrays are steered into a given direction and we may apply some attenuation (tapering) to influence the sidelobe level. The sum of the subarrays then gives the sum beam output. The subarrays can be viewed as a superarray with elements having different patterns steered into the selected direction. The subarrays should have unequal size and shape to avoid grating effects for subsequent array processing, because the subarray centers constitute a sparse array (for details, see [6]). 


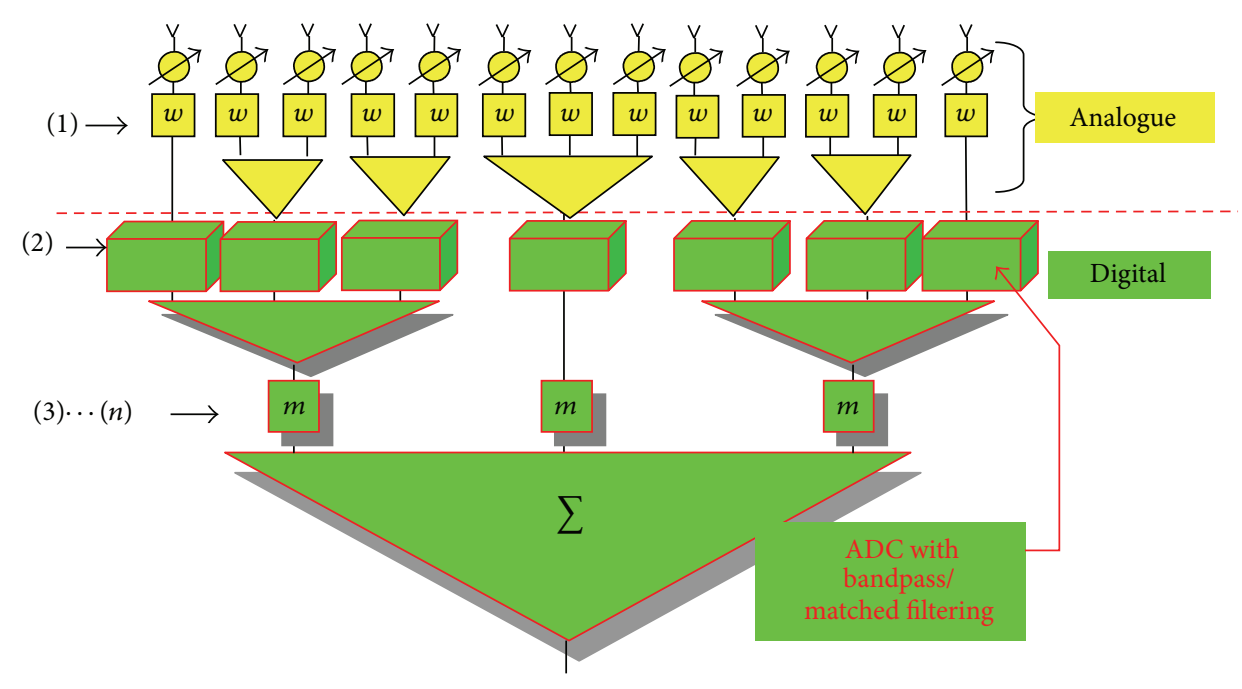

FIGURE 1: Principle of forming subarrays.

The principle is indicated in Figure 1, and properties and options are described in $[6,7]$. In particular, one can also combine new subarrays at the digital level or distribute the desired tapering over the analog level (1) and various digital levels $(2) \cdots(n)$.

Beamforming using subarrays can be mathematically described by a simple matrix operation. Let the complex array element outputs be denoted by $\mathbf{z}$. The subarray forming operation is described by a subarray forming matrix $\mathbf{T}$ by which the element outputs are summed up as $\widetilde{\mathbf{z}}=\mathbf{T}^{H} \mathbf{z}$. For $L$ subarrays and $N$ antenna elements, $\mathbf{T}$ is of size $N \times L$. Vectors and matrices at the subarray outputs are denoted by the tilde. Suppose we steer the array into a look direction $\left(u_{0}, v_{0}\right)=$ : $\mathbf{u}_{0}$ by applying phase shifts $a_{i}\left(\mathbf{u}_{0}\right)=e^{j 2 \pi f_{0}\left(x_{i} u_{0}+y_{i} v_{0}\right) / c}$ and apply additional amplitude weighting $g_{i}$ at the elements (real vector of length $N$ ) for a sum beam with low sidelobes, then we have a complex weighting $g_{i} a_{i}\left(\mathbf{u}_{0}\right)$ which can be included in the elements of the matrix $\mathbf{T}$. Here, $f_{0}$ denotes the centre frequency, $x_{i}, y_{i}$ denote the coordinates of the $i$ th array element, $c$ denotes the velocity of light, and $u, v$ denote the components of the unit direction vector in the planar antenna $(x, y)$-coordinate system. The beams are formed digitally with the subarray outputs by applying a final weighting $\widetilde{m}_{i}(i=1, \ldots, L)$ as

$$
y=\widetilde{\mathbf{m}}^{H} \widetilde{\mathbf{z}}
$$

In the simplest case, $\widetilde{\mathbf{m}}$ consists of only ones. The antenna pattern of such a sum beam can then be written as

$$
f(\mathbf{u})=\widetilde{\mathbf{m}}^{H} \mathbf{T}^{H} \mathbf{a}(\mathbf{u})=\widetilde{\mathbf{m}}^{H} \widetilde{\mathbf{a}}(\mathbf{u}),
$$

where $\mathbf{a}(\mathbf{u})=\left(a_{i}(\mathbf{u})\right)_{i=1 \cdots N}$ and $\widetilde{\mathbf{a}}(\mathbf{u})=\mathbf{T}^{H} \mathbf{a}(\mathbf{u})$ denotes the plane wave response at the subarray outputs. All kinds of beams (sum, azimuth and elevation difference, guard channel, etc.) can be formed from these subarray outputs. We can also scan the beam digitally at subarray level into another direction, [7].

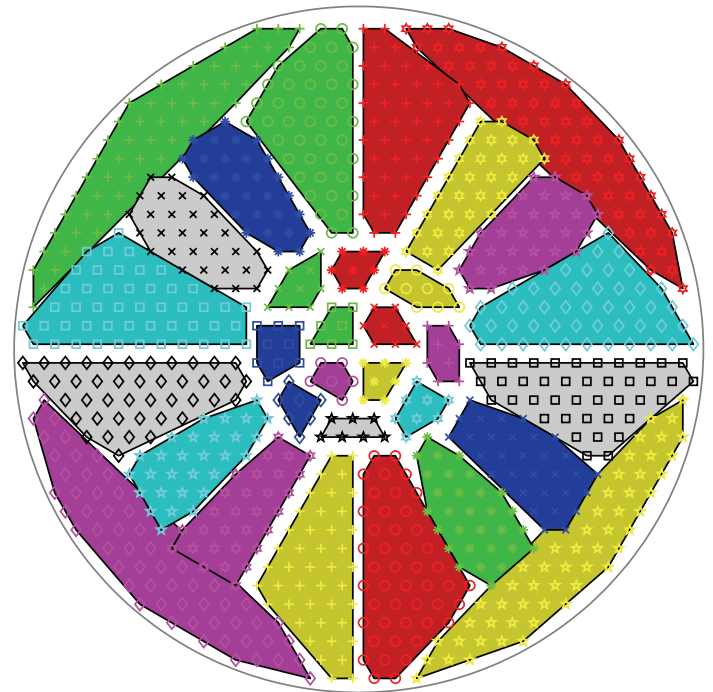

FIGURE 2: 2D generic array with 902 elements grouped into 32 subarrays.

Figure 2 shows a typical planar array with 902 elements on a triangular grid with 32 subarrays. The shape of the subarrays was optimized by the technique of [6] such that the difference beams have low sidelobes when a $-40 \mathrm{~dB}$ Taylor weighting is applied at the elements. We will use this array in the sequel for presenting examples.

An important feature of digital beamforming with subarrays is that the weighting for beamforming can be distributed between the element level (the weighting incorporated in the matrix $\mathbf{T}$ ) and the digital subarray level (the weighting $\widetilde{\mathbf{m}}$ ). This yields some freedom in designing the dynamic range of amplifiers at the elements and the level of the $\mathrm{AD}$-converter input. This freedom also allows to normalize the power of the subarray outputs such that $\mathbf{T}^{H} \mathbf{T}=\mathbf{I}$. As will be shown in Section 4 , this is also a reasonable requirement for adaptive interference suppression to avoid pattern distortions. 


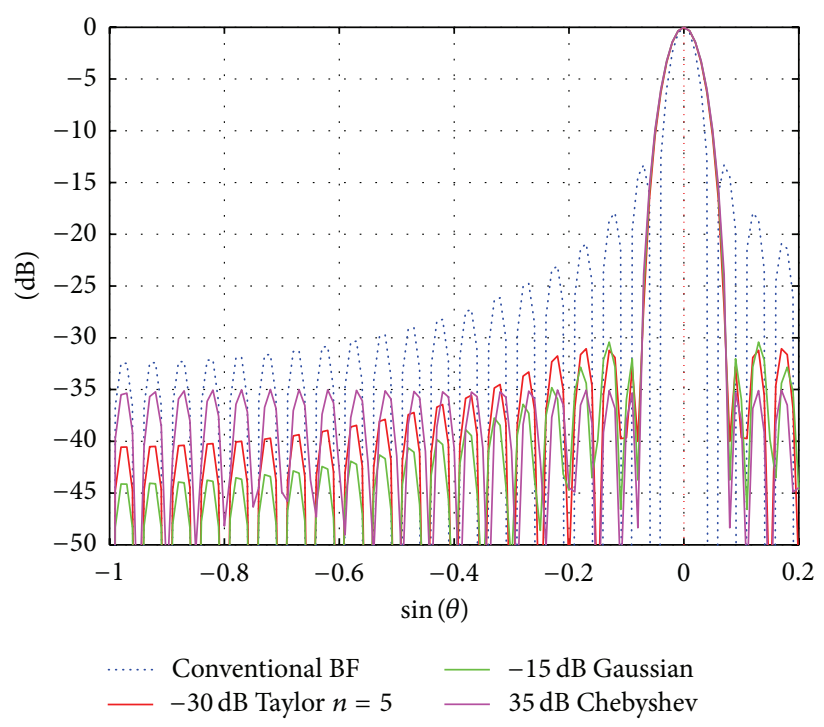

(a) Low sidelobe patterns of equal beamwidth

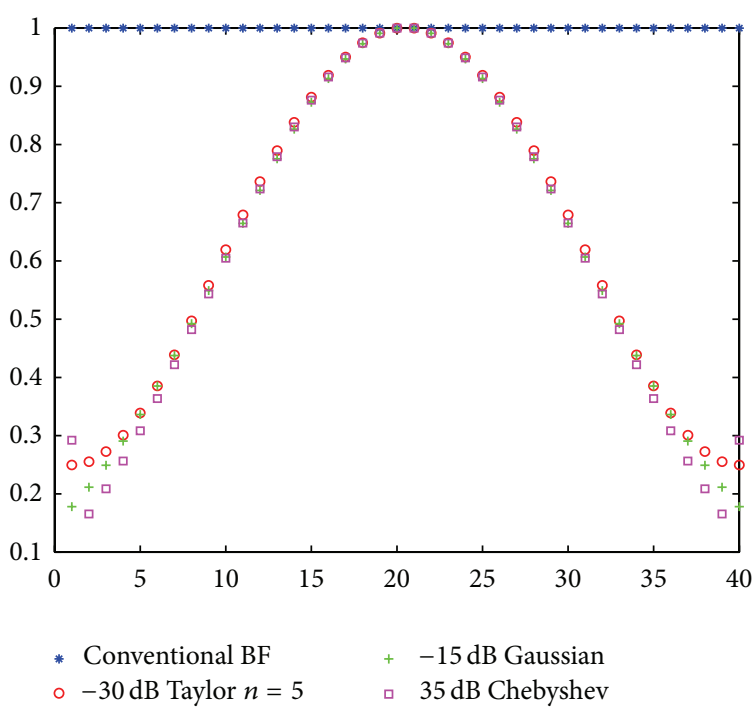

(b) Taper functions for low sidelobes

FIgURE 3: Low sidelobes by amplitude tapering.

2.4. Space-Time Arrays. Coherent processing of a time series $z_{1}, \ldots, z_{K}$ can be written as a beamforming procedure as in (1). For a time series of array snapshots $\mathbf{z}_{1}, \ldots, \mathbf{z}_{K}$, we have therefore a double beamforming procedure of the space-time data matrix $\mathbf{Z}=\left(\mathbf{z}_{1}, \ldots, \mathbf{z}_{K}\right)$ of the form

$$
S=\mathbf{m}_{s}^{H} \mathbf{Z} \mathbf{m}_{t}
$$

where $\mathbf{m}_{s}, \mathbf{m}_{t}$ denote the weight vectors for spatial and temporal beamforming, respectively. Using the rule of Kronecker products, (3) can be written as a single beamforming operation

$$
S=\left(\mathbf{m}_{t} \otimes \mathbf{m}_{s}\right)^{H} \operatorname{vec}\{\mathbf{Z}\},
$$

where $\operatorname{vec}\{\mathbf{Z}\}$ is a vector obtained by stacking all columns of the matrix $\mathbf{Z}$ on top. This shows that mathematically it does not matter whether the data come from spatial or temporal sampling. Coherent processing is in both cases a beamforming-type operation with the correspondingly modified beamforming vector. Relation (4) is often exploited when spatial and temporal parameters are dependent (e.g., direction and Doppler frequency as in airborne radar; see Section 6).

\section{Antenna Pattern Shaping}

Conventional beamforming is the same as coherent integration of the spatially sampled data; that is, the phase differences of a plane wave signal at the array elements are compensated, and all elements are coherently summed up. This results in a pronounced main beam when the phase differences match with the direction of the plane wave and result in sidelobes otherwise. The beam shape and the sidelobes can be influenced by additional amplitude weighting.

Let us consider the complex beamforming weights $w_{i}=$ $g_{i} e^{j 2 \pi f \mathbf{r}_{i}^{T} \mathbf{u} / c}, i=1 \cdots N$. The simplest way of pattern shaping is to impose some bell-shaped amplitude weighting over the aperture like $g_{i}=\cos ^{\nu}\left(\pi x_{i} / A\right)+\alpha$ (for suitable constants $\nu$, $\alpha$ ), or $g_{i}=e^{-v x_{i}^{2}}$. The foundation of these weightings is quite heuristical. The Taylor weighting is optimized in the sense that it leaves the conventional (uniformly weighted) pattern undistorted except for a reduction of the first $n$ sidelobes below a prescribed level. The Dolph-Chebyshev weighting creates a pattern with all sidelobes equal to a prescribed level. Figure 3 shows examples of such patterns for a uniform linear array with 40 elements. The taper functions for low sidelobes were selected such that the $3 \mathrm{~dB}$ beamwidth of all patterns is equal. The conventional pattern is plotted for reference showing how tapering increases the beamwidth. Which of these taperings may be preferred depends on the emphasis on close in and far off sidelobes. Another point of interest is the dynamic range of the weights and the SNR loss, because at the array elements only attenuations can be applied. One can see that the Taylor tapering has the smallest dynamic range. For planar arrays the efficiency of the taperings is slightly different.

The rationale for low sidelobes is that we want to minimize some unknown interference power coming over the sidelobes. This can be achieved by solving the following optimization problem, [8]:

$$
\begin{aligned}
& \min _{\mathbf{w}} \quad \int_{\Omega}\left|\mathbf{w}^{H} \mathbf{a}(\mathbf{u})\right|^{2} p(\mathbf{u}) d \mathbf{u} \\
& \text { subject to } \quad \mathbf{w}^{H} \mathbf{a}_{0}=1 \text {, or equivalently } \\
& \min _{\mathbf{w}} \mathbf{w} \mathbf{C w} \\
& \text { s.t. } \quad \mathbf{w}^{H} \mathbf{a}_{0}=\mathbf{1}, \\
& \text { with } \mathbf{C}=\int_{\Omega} \mathbf{a}(\mathbf{u}) \mathbf{a}(\mathbf{u})^{H} p(\mathbf{u}) d \mathbf{u} .
\end{aligned}
$$



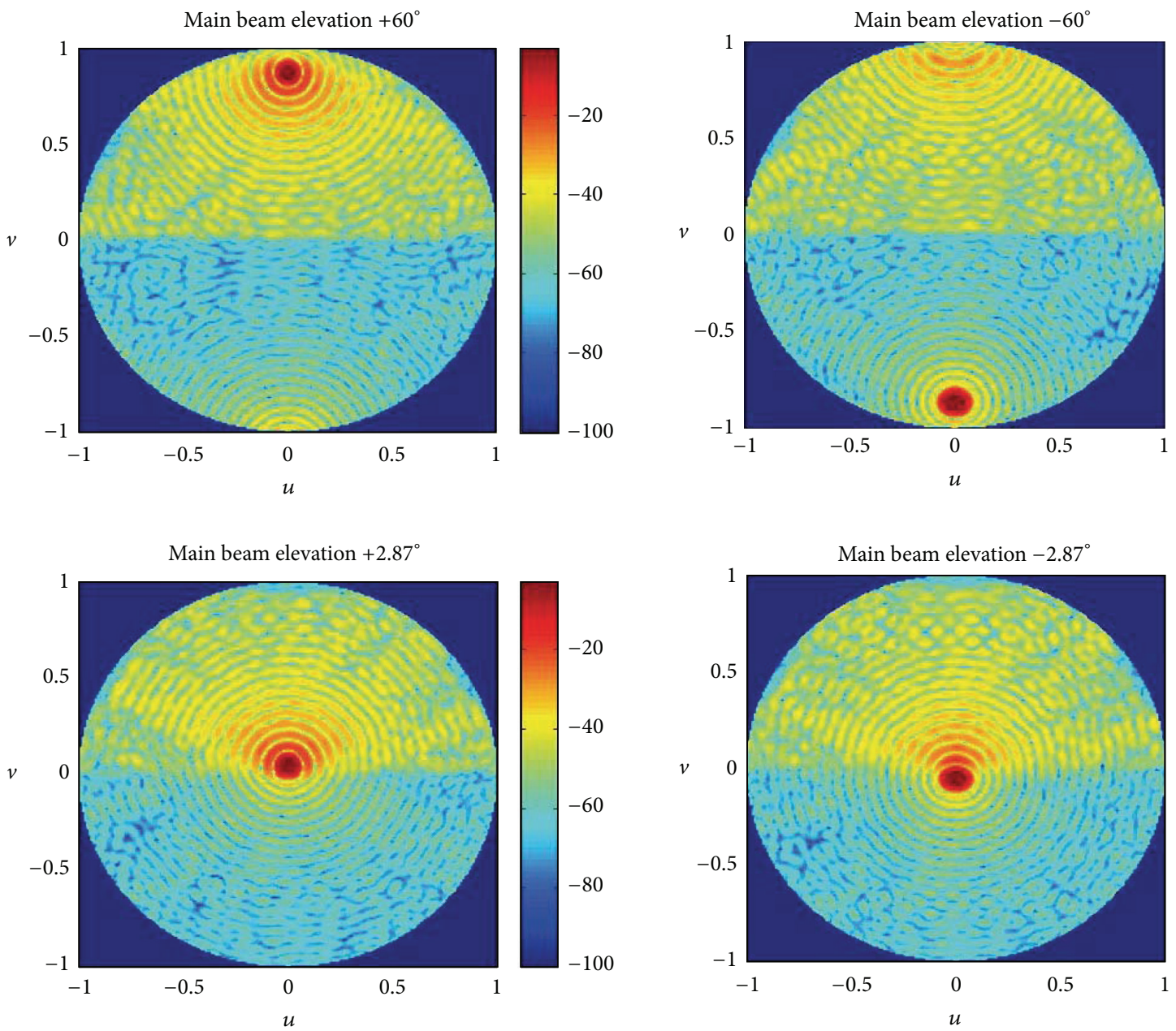

FIGURE 4: Antenna patterns of planar array with reduced sidelobes at lower elevations for different beam pointing directions (by courtesy of W. Bürger of Fraunhofer (FHR)).

$\Omega$ denotes the angular sector where we want to influence the pattern, for example, the whole visible region $u^{2}+v^{2}<1$, and $p$ is a weighting function which allows to put different emphasis on the criterion in different angular regions. The solution of this optimization is

$$
\mathbf{w}=\frac{\mathbf{C}^{-1} \mathbf{a}_{0}}{\mathbf{a}_{0}^{H} \mathbf{C}^{-1} \mathbf{a}_{0}} .
$$

For the choice of the function $p$, we remark that for a global reduction of the sidelobes when $\Omega=\left\{\mathbf{u} \in \mathbb{R}^{2} \mid u^{2}+v^{2} \leq 1\right\}$, one should exclude the main beam from the minimization by setting $p=0$ on this set of directions (in fact, a slightly larger region is recommended, e.g., the null-to-null width) to allow a certain mainbeam broadening. One may also form discrete nulls in directions $\mathbf{u}_{1}, \ldots, \mathbf{u}_{M}$ by setting $p(\mathbf{u})=\sum_{k=1}^{M} \delta(\mathbf{u}-$ $\mathbf{u}_{k}$ ). The solution of (5) then can be shown to be

$$
\begin{gathered}
\mathbf{w}=\frac{\mathbf{P a}_{0}}{\mathbf{a}_{0}^{H} \mathbf{P} \mathbf{a}_{0}} \quad \text { with } \mathbf{P}=\mathbf{I}-\mathbf{A}\left(\mathbf{A}^{H} \mathbf{A}\right)^{-1} \mathbf{A}^{H}, \\
\mathbf{A}=\left(\mathbf{a}\left(\mathbf{u}_{1}\right), \ldots, \mathbf{a}\left(\mathbf{u}_{M}\right)\right) .
\end{gathered}
$$

This is just the weight for deterministic nulling. To avoid insufficient suppression due to channel inaccuracies, one may also create small extended nulls using the matrix $\mathbf{C}$. The form of these weights shows the close relationship to the adaptive beamforming weights in (11) and (17).

An example for reducing the sidelobes in selected areas where interference is expected is shown in Figure 4. This is an application from an airborne radar where the sidelobes in the negative elevation space have been lowered to reduce ground clutter.

\section{Adaptive Interference Suppression}

Deterministic pattern shaping is applied if we have rough knowledge about the interference angular distribution. In the sidelobe region, this method can be inefficient because the antenna response to a plane wave (the vector $\mathbf{a}(\mathbf{u})$ ) must be exactly known which is in reality seldom the case. Typically, much more suppression is applied than necessary with the price paid by the related beam broadening and SNR loss. Adaptive interference suppression needs no knowledge of the directional behavior and suppresses the interference only as 
much as necessary. The proposition for this approach is that we are able to measure or learn in some way the adaptive beamforming $(\mathrm{ABF})$ weights.

In the sequel, we formulate the $\mathrm{ABF}$ algorithms for subarray outputs as described in (1). This includes element space ABF for subarrays containing only one element.

4.1. Adaptive Beamforming Algorithms. Let us first suppose that we know the interference situation; that is, we know the interference covariance matrix $\mathbf{Q}$. What is the optimum beamforming vector $\mathbf{w}$ ? From the Likelihood Ratio test criterion, we know that the probability of detection is maximized if we choose the weight vector that maximizes the signal-tonoise-plus-interference ratio (SNIR) for a given (expected) signal $\mathbf{a}_{0}$,

$$
\max _{\mathbf{w}} \frac{\left|\mathbf{w}^{H} \mathbf{a}_{0}\right|^{2}}{E\left\{\left|\mathbf{w}^{H} \mathbf{n}\right|^{2}\right\}}=\max _{\mathbf{w}} \frac{\left|\mathbf{w}^{H} \mathbf{a}_{0}\right|^{2}}{\mathbf{w}^{H} \mathbf{Q w}} .
$$

The solution of this optimization is

$$
\mathbf{w}=\mu \mathbf{Q}^{-1} \mathbf{a}_{0} \quad \text { with } \mathbf{Q}=E\left\{\mathbf{n} \mathbf{n}^{H}\right\}
$$

$\mu$ is a free normalization constant and $\mathbf{n}$ denotes interference and receiver noise. This weighting has a very intuitive interpretation. If we decompose $\mathbf{Q}^{-1}=\mathbf{L} \mathbf{L}^{H}$ and apply this weight to the data, we have $\mathbf{w}^{H} \mathbf{z}=\mathbf{a}_{0}^{H} \mathbf{Q}^{-1} \mathbf{z}=\mathbf{a}_{0}^{H} \mathbf{L}^{H} \mathbf{L z}=\left(\mathbf{L} \mathbf{a}_{0}\right)^{H}(\mathbf{L z})$. This reveals that $\mathrm{ABF}$ does nothing else but a pre-whiten and match operation: if $\mathbf{z}$ contains only interference, that is, $E\left\{\mathbf{z z}^{H}\right\}=\mathbf{Q}$, then $E\left\{(\mathbf{L z})(\mathbf{L z})^{H}\right\}=\mathbf{I}$, the prewhitening operation; the operation of $\mathbf{L}$ on the (matched) signal vector $\mathbf{a}_{0}$ restores just the matching necessary with the distortion from the prewhitening operation.

This formulation for weight vectors applied at the array elements can be easily extended to subarrays with digital outputs. As mentioned in Section 2.3, a subarrayed array can be viewed as a superarray with directive elements positioned at the centers of the subarrays. This means that we have only to replace the quantities $\mathbf{a}, \mathbf{n}$ by $\widetilde{\mathbf{a}}=\mathbf{T}^{H} \mathbf{a}, \widetilde{\mathbf{n}}=\mathbf{T}^{H} \mathbf{n}$. However, there is a difference with respect to receiver noise. If the noise at the elements is white with covariance matrix $\sigma^{2} \mathbf{I}$ it will be at subarray outputs with covariance matrix $\widetilde{\mathbf{Q}}=$ $\sigma^{2} \mathbf{T}^{H} \mathbf{T}$. Adaptive processing will turn this into white noise. Furthermore, if we apply at the elements some weighting for low sidelobes, which are contained in the matrix $\mathbf{T}, \mathrm{ABF}$ will reverse this operation by the pre-whiten and match principle and will distort the low sidelobe pattern. This can be avoided by normalizing the matrix $\mathbf{T}$ such that $\mathbf{T} \mathbf{T}^{H}=\mathbf{I}$. This can be achieved by normalizing the element weight as mentioned in Section 2.3 (for nonoverlapping subarrays).

Sometimes interference suppression is realized by minimizing only the jamming power subject to additional constraints, for example, $\mathbf{w}^{H} \mathbf{c}_{i}=k_{i}$, for suitable vectors $\mathbf{c}_{i}$ and numbers $k_{i}, i=1 \cdots r$. Although this is an intuitively reasonable criterion, it does not necessarily give the maximum SNIR. For certain constraints however both solutions are equivalent. The constrained optimization problem can be written in general terms as

$$
\begin{array}{ll}
\min _{\mathbf{w}} & \mathbf{w}^{H} \mathbf{Q} \mathbf{w} \\
\text { s.t. } & \mathbf{w}^{H} \mathbf{C}=\mathbf{k} \quad\left(\text { or } \mathbf{w}^{H} \mathbf{c}_{i}=k_{i}, i=1 \cdots r\right),
\end{array}
$$

and it has the solution

$$
\mathbf{w}=\sum_{i=1}^{r} \lambda_{i} \mathbf{Q}^{-1} \mathbf{c}_{i}=\mathbf{Q}^{-1} \mathbf{C}\left(\mathbf{C}^{H} \mathbf{Q}^{-1} \mathbf{C}\right)^{-1} \mathbf{k} .
$$

Examples of special cases are as follows:

(i) Single unit gain directional constraint: $\mathbf{w}^{H} \mathbf{a}_{0}=1 \Rightarrow$ $\mathbf{w}=\left(\mathbf{a}_{0}^{H} \mathbf{Q}^{-1} \mathbf{a}_{0}\right)^{-1} \mathbf{Q}^{-1} \mathbf{a}_{0}$. This is obviously equivalent to the SNIR-optimum solution (9) with a specific normalization.

(ii) Gain and derivative constraint: $\mathbf{w}^{H} \mathbf{a}_{0}=1, \mathbf{w}^{H} \mathbf{a}_{0}^{\prime}=$ $0 \Rightarrow \mathbf{w}=\mu \mathbf{Q}^{-1} \mathbf{a}_{0}+\kappa \mathbf{Q}^{-1} \mathbf{a}_{0}^{\prime}$ with suitable values of the Lagrange parameters $\mu, \lambda$. A derivative constraint is added to make the weight less sensitive against mismatch of the steering direction.

(iii) Gain and norm constraint: $\mathbf{w}^{H} \mathbf{a}_{0}=1, \mathbf{w}^{H} \mathbf{w}=c \Rightarrow$ $\mathbf{w}=\mu(\mathbf{Q}+\delta \mathbf{I})^{-1} \mathbf{a}_{0}$. The norm constraint is added to make the weight numerically stable. This is equivalent to the famous diagonal loading technique which we will consider later.

(iv) Norm constraint only: $\mathbf{w}^{H} \mathbf{w}=1 \Rightarrow \mathbf{w}=\min E V(\mathbf{Q})$. Without a directional constraint the weight vector produces a nearly omnidirectional pattern, but with nulls in the interference directions. This is also called the power inversion weight, because the pattern displays the inverted interference power.

As we mentioned before, fulfilling the constraints may imply a loss in SNIR. Therefore, several techniques have been proposed to mitigate the loss. The first idea is to allow a compromise between power minimization and constraints by introducing coupling factors $b_{i}$ and solve a soft constraint optimization

$$
\begin{array}{ll}
\min _{\mathbf{w}} & \mathbf{w}^{H} \mathbf{Q w}+\sum_{i=1}^{r} b_{i}\left|\mathbf{w}^{H} \mathbf{c}_{i}-k_{i}\right|^{2} \text { or } \\
\min _{\mathbf{w}} & \mathbf{w}^{H} \mathbf{Q w}+\left(\mathbf{w}^{H} \mathbf{C}-\mathbf{k}\right)^{H} \mathbf{B}\left(\mathbf{w}^{H} \mathbf{C}-\mathbf{k}\right)
\end{array}
$$

with $\mathbf{B}=\operatorname{diag}\left\{b_{1}, \ldots, b_{r}\right\}$. The solution of the soft-constraint optimization is

$$
\mathbf{w}=\left(\mathbf{Q}+\mathbf{C B C}^{H}\right)^{-1} \mathbf{C B k} .
$$

One may extend the constrained optimization by adding inequality constraints. This leads to additional and improved robustness properties. A number of methods of this kind have been proposed, for example, in [9-12]. As we are only presenting the principles here we do not go into further details. 


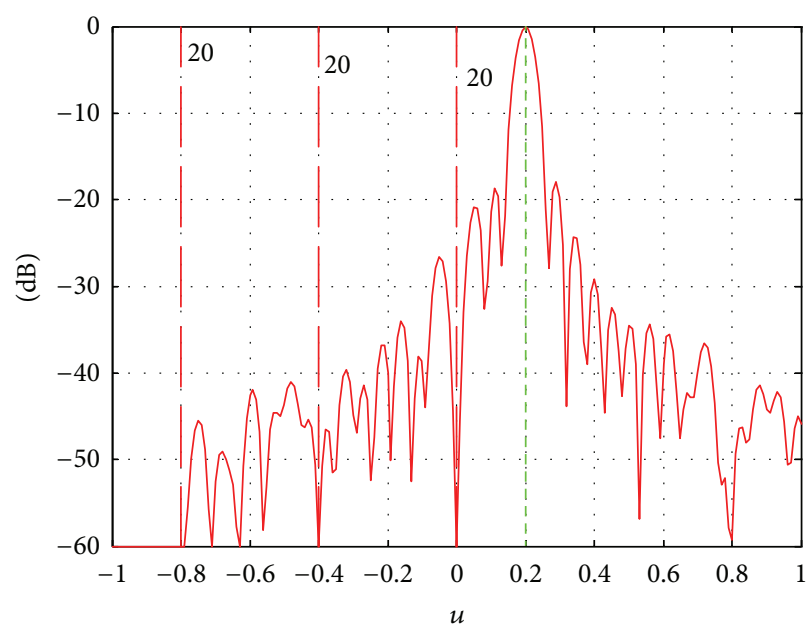

(a) Adapted antenna pattern

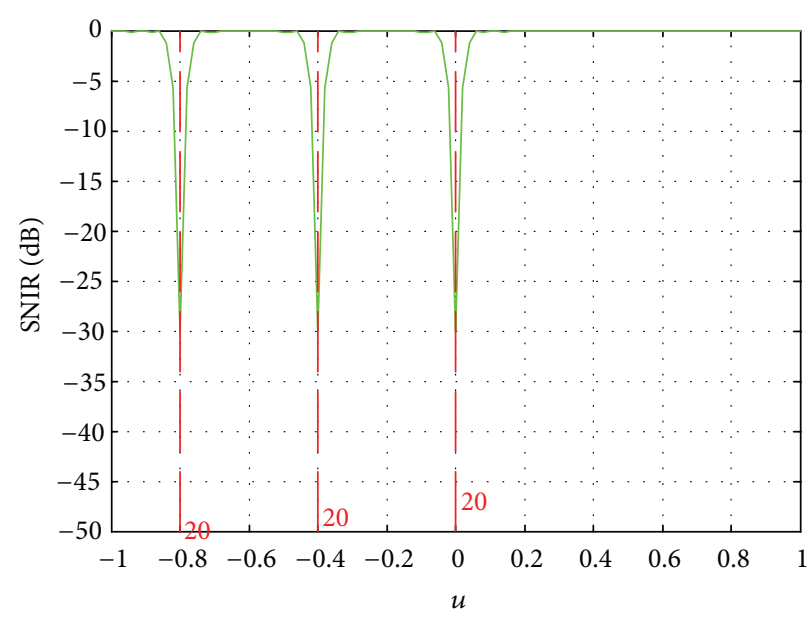

(b) SNIR

FIGURE 5: Antenna and normalized SNIR patterns for a three jammer configuration and generic array.

The performance of $\mathrm{ABF}$ is often displayed by the adapted antenna pattern. A typical adapted antenna pattern with 3 jammers of $20 \mathrm{~dB}$ SNR is shown in Figure 5(a) for generic array of Figure 2. This pattern does not show how the actual jamming power and the null depth play together.

Plots of the SNIR are better suited for displaying this effect. The SNIR is typically plotted for varying target direction while the interference scenario is held fixed, as seen in Figure 5(b). The SNIR is normalized to the SNR in the clear absence of any jamming and without $\mathrm{ABF}$. In other words, this pattern shows the insertion loss arising from the jamming scenario with applied ABF.

The effect of target and steering direction mismatch is not accounted for in the SNIR plot. This effect is displayed by the scan pattern, that is, the pattern that arises if the adapted beam scans over a fixed target and interference scenario. Such a plot is rarely shown because of the many parameters to be varied. In this context, we note that for the case that the training data contains the interference and noise alone the main beam of the adapted pattern is fairly broad similar to the unadapted sum beam and is therefore fairly insensitive to pointing mismatch. How to obtain an interference-alone covariance matrix is a matter of proper selection of the training data as mentioned in the following section.

Figure 5 shows the case of an untapered planar antenna. The first sidelobes of the unadapted antenna pattern are at $-17 \mathrm{~dB}$ and are nearly unaffected by the adaptation process. If we have an antenna with low sidelobes, the peak sidelobe level is much more affected; see Figure 6. Due to the tapering we have a loss in SNIR of $1.7 \mathrm{~dB}$ compared to the reference antenna (untapered without $\mathrm{ABF}$ and jamming).

4.2. Estimation of Adaptive Weights. In reality, the interference covariance matrix is not known and must be estimated from some training data $\mathbf{Z}=\left(\mathbf{z}_{1}, \ldots, \mathbf{z}_{K}\right)$. To avoid signal cancellation, the training data should only contain the interference alone. If we have a continuously emitting interference source (noise jammer) one may sample immediately after or before the transmit pulse (leading or rear dead zone). On the other hand, if we sample the training data before pulse compression the desired signal is typically much below the interference level, and signal cancellation is negligible. Other techniques are described in [13]. The maximum likelihood estimate of the covariance matrix is then

$$
\widehat{\mathbf{Q}}_{\mathrm{SMI}}=\frac{1}{K} \sum_{k=1}^{K} \mathbf{z}_{k} \mathbf{z}_{k}^{H} .
$$

This is called the Sample Matrix Inversion algorithm (SMI). The SMI method is only asymptotically a good estimate. For small sample size, it is known to be not very stable. For matrix invertibility, we need at least $K=N$ samples. According to Brennan's Rule, for example, [1], one needs $2 K$ samples to obtain an average loss in SNIR below $3 \mathrm{~dB}$. For smaller sample size, the performance can be considerably worse. However, by simply adding a multiple of the identity matrix to the SMI estimate, a close to optimum performance can be achieved. This is called the loaded sample matrix estimate (LSMI)

$$
\widehat{\mathbf{Q}}_{\mathrm{LSMI}}=\frac{1}{K} \sum_{k=1}^{K} \mathbf{z}_{k} \mathbf{z}_{k}^{H}+\delta \cdot \mathbf{I} .
$$

The drastic difference between SMI and LSMI is shown in Figure 7 for the planar array of Figure 2 for three jammers of $20 \mathrm{~dB}$ input JNR with 32 subarrays and only 32 data snapshots. For a "reasonable" choice of the loading factor (a rule of thumb is $\delta=2 \sigma^{2} \cdots 4 \sigma^{2}$ for an untapered antenna) we need only $2 M$ snapshots to obtain a $3 \mathrm{~dB}$ SNIR loss, if $M$ denotes the number of jammers (dominant eigenvalues) present, [13]. So the sample size can be considerably lower than the dimension of the matrix. The effect of the loading factor is that the dynamic range of the small eigenvalues is compressed. The small eigenvalues possess the largest statistical fluctuation but have the greatest influence on the weight fluctuation due to the matrix inversion. 


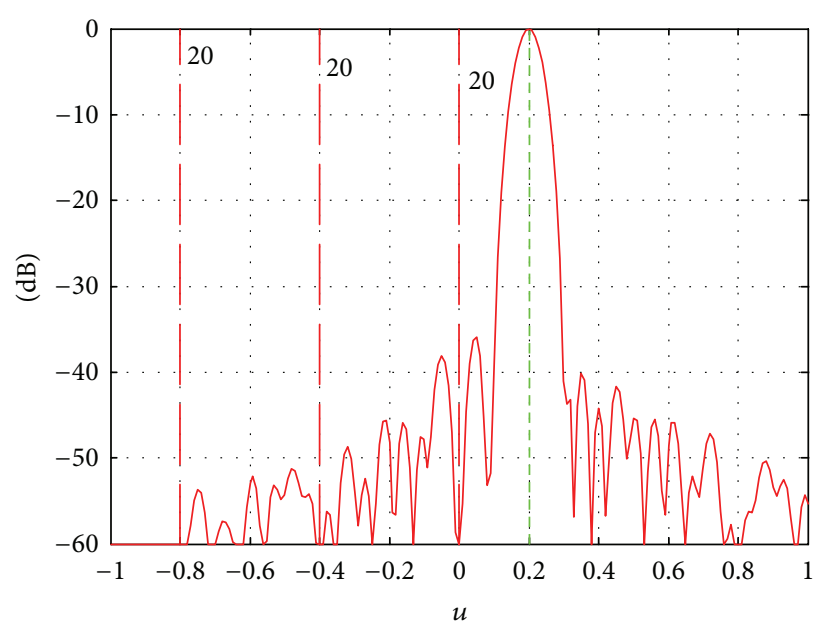

(a) Adapted antenna pattern

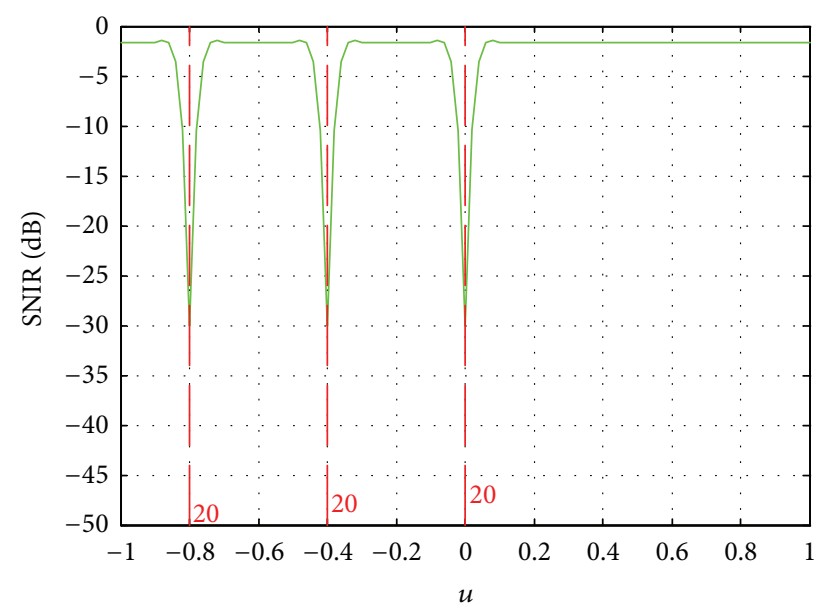

(b) SNIR

FIGURE 6: Antenna and normalized SNIR patterns for a three jammer configuration for antenna with low sidelobes (-40 dB Taylor weighting).

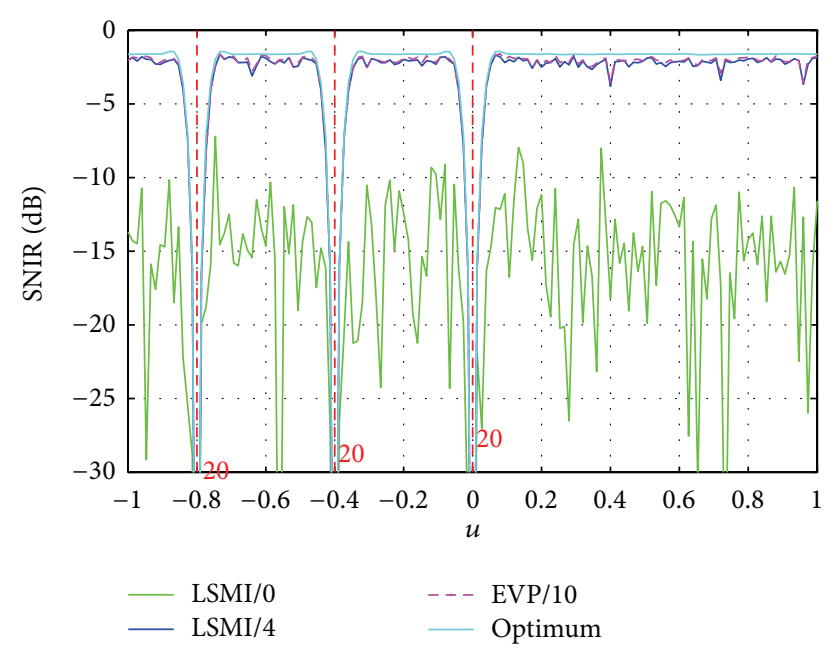

FIGURE 7: SNIR for SMI, LSMI $\left(\delta=4 \sigma^{2}\right)$, and eigenvector projection with $\operatorname{dim}($ JSS $)=3$.

One may go even further and ignore the small eigenvalue estimates completely; that is, one tries to find an estimate of the inverse covariance matrix based on the dominant eigenvectors and eigenvalues. For high SNR, we can replace the inverse covariance matrix by a projection matrix. Suppose we have $M$ jammers with amplitudes $b_{1}(t), \ldots, b_{M}(t)$ in directions $\mathbf{u}_{1}, \ldots, \mathbf{u}_{M}$. If the received data has the form $\mathbf{z}\left(t_{k}\right)=$ $\sum_{m=1}^{M} \mathbf{a}\left(\mathbf{u}_{m}\right) b_{m}\left(t_{k}\right)+\mathbf{n}\left(t_{k}\right)$, or short $\mathbf{z}_{k}=\mathbf{A} \mathbf{b}_{k}+\mathbf{n}_{k}$, then

$$
E\left\{\mathbf{z z}^{H}\right\}=\mathbf{Q}=\mathbf{A} \mathbf{B} \mathbf{A}^{H}+\mathbf{I} .
$$

Here, we have normalized the noise power to 1 and $\mathbf{B}=$ $E\left\{\mathbf{b} \mathbf{b}^{H}\right\}$. Using the matrix inversion lemma, we have

$$
\begin{aligned}
\mathbf{Q}^{-1} & =\mathbf{I}-\mathbf{A}\left(\mathbf{B}^{-1}+\mathbf{A}^{H} \mathbf{A}\right)^{-1} \mathbf{A}^{H} \\
& \underset{\mathbf{B} \rightarrow \infty}{\longrightarrow} \mathbf{I}-\mathbf{A}\left(\mathbf{A}^{H} \mathbf{A}\right)^{-1} \mathbf{A}^{H}=\mathbf{P}_{\mathbf{A}}^{\perp}
\end{aligned}
$$

$\mathbf{P}_{\mathbf{A}}^{\perp}$ is a projection on the space orthogonal to the columns of $\mathbf{A}$. For strong jammers, the space spanned by the columns of $\mathbf{A}$ will be the same as the space spanned by the dominant eigenvectors. We may therefore replace the estimated inverse covariance matrix by a projection on the complement of the dominant eigenvectors. This is called the EVP method. As the eigenvectors $\mathbf{X}$ are orthonormalized, the projection can be written as $\mathbf{P}_{X}^{\perp}=\mathbf{I}-\mathbf{X} \mathbf{X}^{H}$.

Figure 7 shows the performance of the EVP method in comparison with SMI, LSMI. Note the little difference between LSMI and EVP. The results with the three methods are based on the same realization of the covariance estimate.

For EVP, we have to know the dimension of the jammer subspace (dimJSS). In complicated scenarios and with channel errors present, this value can be difficult to determine. If dimJSS is grossly overestimated, a loss in SNIR occurs. If dimJSS is underestimated the jammers are not fully suppressed. One is therefore interested in subspace methods with low sensitivity against the choice of the subspace dimension. This property is achieved by a "weighted projection," that is, by replacing the projection by

$$
\mathbf{P}_{\mathrm{LMI}}=\mathbf{I}-\mathbf{X D X}^{H}
$$

where $\mathbf{D}$ is a diagonal weighting matrix and $\mathbf{X}$ is a set orthonormal vectors spanning the interference subspace. $\mathbf{P}_{\text {LMI }}$ does not have the mathematical properties of a projection. Methods of this type of are called lean matrix inversion (LMI). A number of methods have been proposed that can be interpreted as an LMI method with different weighting matrices $\mathbf{D}$. The LMI matrix can also be economically calculated by an eigenvector-free QR-decomposition method, [14].

One of the most efficient methods for pattern stabilization while maintaining a low desired sidelobe level is the constrained adaptive pattern synthesis (CAPS) algorithm, [15], which is also a subspace method. Let $\mathbf{m}$ be the vector for 
beamforming with low sidelobes in a certain direction. In full generality, the CAPS weight can be written as

$$
\begin{aligned}
\mathbf{w}_{\mathrm{CAPS}}= & \frac{1}{\mathbf{m}^{H} \widehat{\mathbf{R}}^{-1} \mathbf{m}} \widehat{\mathbf{Q}}_{\mathrm{SMI}}^{-1} \mathbf{m}-\mathbf{X}_{\perp}\left(\mathbf{X}_{\perp}^{H} \mathbf{C X}_{\perp}\right)^{-1} \\
& \times \mathbf{X}_{\perp}^{H} \mathbf{C}\left(\frac{1}{\mathbf{m}^{H} \widehat{\mathbf{R}}^{-1} \mathbf{m}} \widehat{\mathbf{Q}}_{\mathrm{SMI}}^{-1} \mathbf{m}-\mathbf{m}\right),
\end{aligned}
$$

where the columns of the matrix $\mathbf{X}_{\perp}$ span the space orthogonal to $[\mathbf{X}, \mathbf{m}]$ and $\mathbf{X}$ is again a unitary $L \times M$ matrix with columns spanning the interference subspace which is assumed to be of dimension $M$. C is a directional weighting matrix, $\mathbf{C}=\int_{\Omega} \mathbf{a}(\mathbf{u}) \mathbf{a}(\mathbf{u})^{H} p(\mathbf{u}) d \mathbf{u}, \Omega$ denotes the set of directions of interest, and $p(\mathbf{u})$ is a directional weighting function. If we use no directional weighting, $\mathbf{C} \approx \mathbf{I}$, the CAPS weight vector simplifies to

$$
\mathbf{w}_{\mathrm{CAPS}}=\mathbf{m}+\mathbf{P}_{[\mathbf{X}, \mathbf{m}]}\left(\frac{1}{\mathbf{m}^{H} \widehat{\mathbf{R}}^{-1} \mathbf{m}} \widehat{\mathbf{Q}}_{\mathrm{SMI}}^{-1} \mathbf{m}-\mathbf{m}\right),
$$

where $\mathbf{P}_{[\mathbf{X}, \mathbf{m}]}$ denotes the projection onto the space spanned by the columns of $\mathbf{X}$ and $\mathbf{m}$.

\subsection{Determination of the Dimension of Jammer Subspace} (dimJSS). Subspace methods require an estimate of the dimension of the interference subspace. Usually this is derived from the sample eigenvalues. For complicated scenarios and small sample size, a clear decision of what constitutes a dominant eigenvalue may be difficult. There are two principle approaches to determine the number of dominant eigenvalues, information theoretic criteria and noise power tests.

The information theoretic criteria are often based on the sphericity test criterion; see, for example, [16],

$$
T(m)=\frac{(1 /(N-m)) \sum_{i=m+1}^{N} \lambda_{i}}{\left(\prod_{i=m+1}^{N} \lambda_{i}\right)^{1 /(N-m)}},
$$

where $\lambda_{i}$ denote the eigenvalues of the estimated covariance matrix ordered in decreasing magnitude. The ratio of the arithmetic to geometric mean of the eigenvalues is a measure of the equality of the eigenvalues. The information theoretic criteria minimize this ratio with a penalty function added; for example, the Akaike Information Criterion (AIC) and Minimum Description Length (MDL) choose $\operatorname{dim} J S S \widehat{M}$ as the minimum of the following functions:

$$
\begin{gathered}
\operatorname{AIC}(m)=K(N-m) \log [T(m)]+m(2 N-m) \\
\operatorname{MDL}(m)=K(N-m) \log [T(m)]+\left(\frac{m}{2}\right)(2 N-m) \log K .
\end{gathered}
$$

The noise power threshold tests (WNT) assume that the noise power $\sigma^{2}$ is known and just check the estimated noise power against this value, [16]. This leads to the statistic

$$
L(m)=\frac{2 K}{\sigma^{2}} \sum_{i=m+1}^{N} \lambda_{i}
$$

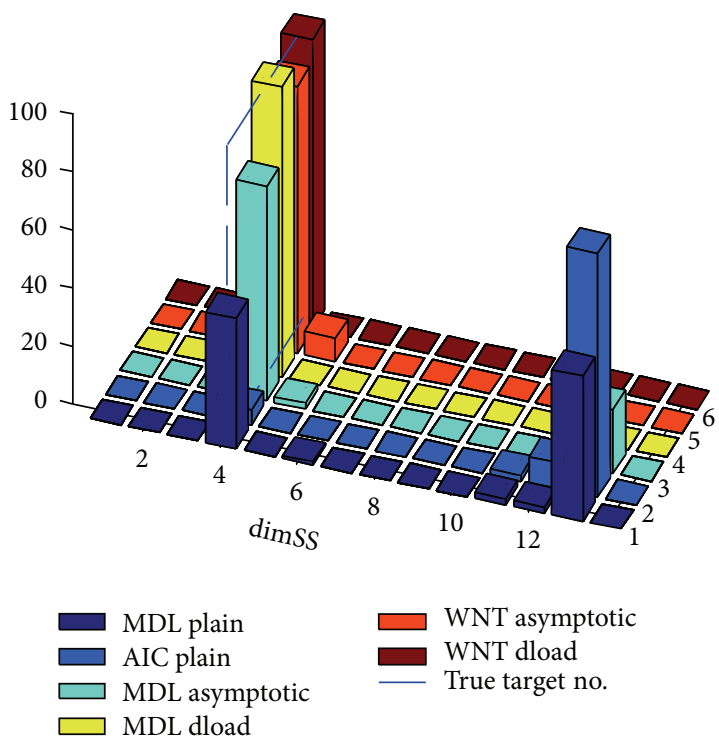

FIGURE 8: Comparison of tests for linear array with $N=14$ elements, $K=14$ snapshots, with and without asymptotic correction or diagonal loading (dload) of $1 \sigma^{2}$.

and the decision is found if the test statistic is for the first time below the threshold:

$$
\begin{aligned}
& \text { for } i=1 \cdots N \text { do } \\
& \quad \text { if } L(m) \leq \chi_{2 K(N-m) ; \alpha}^{2}: \widehat{M}=m \text {; STOP; }
\end{aligned}
$$

end.

The symbol $\chi_{r ; \alpha}^{2}$ denotes the $\alpha$-percentage point of the $\chi^{2}$ distribution with $r$ degrees of freedom. The probability to overestimate $\operatorname{dim}$ JSS is then asymptotically bounded by $\alpha$. More modern versions of this test have been derived, for example, [17].

For small sample size, AIC and MDL are known for grossly overestimating the number of sources. In addition, bandwidth and array channels errors lead to a leakage of the dominant eigenvalues into the small eigenvalues, [18]. Improved eigenvalue estimates for small sample size can mitigate this effect. The simplest way could be to use the asymptotic approximation using the well-known linkage factors, [19],

$$
\widehat{\hat{\lambda}}_{i}=\widehat{\lambda}_{i}-\frac{1}{K} \widehat{\lambda}_{i} \sum_{\substack{j=1 \\ j \neq i}}^{N} \frac{\hat{\lambda}_{i}}{\widehat{\lambda}_{i}-\widehat{\lambda}_{j}} .
$$

More refined methods are also possible; see [16]. However, as explained in [16], simple diagonal loading can improve AIC and MDL for small sample size and make these criteria robust against errors. For the WNT this loading is contained in the setting of the assumed noise level $\sigma^{2}$. Figure 8 shows an example of a comparison of MDL and AIC without any corrections, MDL and WNT with asymptotic correction (25), and MDL and WNT with diagonal loading of $\mu=1 \sigma^{2}$. The 
threshold for WNT was set for a probability to overestimate the target number of $\alpha=10 \%$. The scenario consists of four sources at $u=-0.7,-0.55,-0.31,-0.24$ with SNR of $18,6,20$, $20.4 \mathrm{~dB}$ and a uniform linear antenna with 14 elements and $10 \%$ relative bandwidth leading to some eigenvalue leakage. Empirical probabilities were determined from 100 Monte Carlo trials. Note that the asymptotic correction seems to work better for WNT than for MDL. With diagonal loading, all decisions with both MDL and WNT were correct (equal to 4$)$.

A more thorough study of the small sample size dimJSS estimation problem considering the "effective number of identifiable signals" has been performed in [20], and a new modified information theoretic criterion has been derived.

\section{Parameter Estimation and Superresolution}

The objective of radar processing is not to maximize the SNR but to detect targets and determine their parameters. For detection, the SNR is a sufficient statistic (for the likelihood ratio test); that is, if we maximize the SNR we maximize also the probability of detection. Only for these detected targets we have then a subsequent procedure to estimate the target parameters: direction, range, and possibly Doppler. Standard radar processing can be traced back to maximum likelihood estimation of a single target which leads to the matched filter, [21]. The properties of the matched filter can be judged by the beam shape (for angle estimation) and by the ambiguity function (for range and Doppler estimation). If the ambiguity function has a narrow beam and sufficiently low sidelobes, the model of a single target is a good approximation as other targets are attenuated by the sidelobes. However, if we have closely spaced targets or high sidelobes, multiple target models have to be used for parameter estimation. A variety of such estimation methods have been introduced which we term here "superresolution methods." Historically, these methods have often been introduced to improve the limited resolution of the matched filter.

5.1. Superresolution. The resolution limit for classical beamforming is the $3 \mathrm{~dB}$ beamwidth. An antenna array provides spatial samples of the impinging wavefronts, and one may define a multitarget model for this case. This opens the possibility for enhanced resolution. These methods have been discussed since decades, and textbooks on this topic are available, for example, [22]. We formulate here the angle parameter estimation problem (spatial domain), but corresponding versions can be applied in the time domain as well. In the spatial domain, we are faced with the typical problems of irregular sampling and subarray processing.

From the many proposed methods, we mention here only some classical methods to show the connections and relationships. We have spectral methods which generate a spiky estimate of the angular spectral density like.

Capon's method:

$$
S_{C}(\mathbf{u})=\left(\mathbf{a}(\mathbf{u})^{H} \widehat{\mathbf{R}}_{\mathrm{ML}}^{-1} \mathbf{a}(\mathbf{u})\right)^{-1} \quad \text { with } \widehat{\mathbf{R}}_{\mathrm{ML}}=\frac{1}{K} \sum_{k=1}^{K} \mathbf{z}_{k} \mathbf{z}_{k}^{H},
$$

and MUSIC method (Multiple Signal Classification):

$$
S_{\text {MUSIC }}(\mathbf{u})=\left(\mathbf{a}(\mathbf{u})^{H} \mathbf{P}^{\perp} \mathbf{a}(\mathbf{u})\right)^{-1},
$$

with $\mathbf{P}^{\perp}=\mathbf{I}-\mathbf{X} \mathbf{X}^{H}$, and $\mathbf{X}$ spanning the dominant subspace. An LMI-version instead of MUSIC would also be possible. The target directions are then found by the $M$ highest maxima of these spectra ( $M$ 1- or 2-dimensional maximizations).

An alternative group of methods are parametric methods, which deliver only a set of "optimal" parameter estimates which explain in a sense the data for the inserted model by $M$ or $2 M$ dimensional optimization [21].

Deterministic ML method (complex amplitudes are assumed deterministic):

$$
\begin{gathered}
F_{\text {det }}(\boldsymbol{\theta})=\operatorname{tr}\left(\mathbf{P}_{\mathbf{A}}^{\perp} \widehat{\mathbf{R}}_{\mathrm{ML}}\right), \quad \text { with } \mathbf{P}_{\mathbf{A}}^{\perp}=\mathbf{I}-\mathbf{A}\left(\mathbf{A}^{H} \mathbf{A}\right)^{-1} \mathbf{A}^{H}, \\
\mathbf{A}=\left(\mathbf{a}\left(\mathbf{u}_{1}\right), \ldots, \mathbf{a}\left(\mathbf{u}_{M}\right)\right) .
\end{gathered}
$$

Stochastic ML method (complex amplitudes are complex Gaussian):

$$
F_{\text {sto }}(\boldsymbol{\theta})=\log \operatorname{det}(\mathbf{R}(\boldsymbol{\theta}))+\operatorname{tr}\left(\mathbf{R}(\boldsymbol{\theta})^{-1} \widehat{\mathbf{R}}_{\mathrm{ML}}\right) \text {, }
$$

where $\mathbf{R}(\boldsymbol{\theta})$ denotes the completely parameterized covariance matrix. A formulation with the unknown directions as the only parameters can be given as

$$
\begin{gathered}
F_{\text {sto }}(\boldsymbol{\theta})=\operatorname{det}\left\{\mathbf{A}(\boldsymbol{\theta}) \mathbf{B}(\boldsymbol{\theta}) \mathbf{A}^{H}(\boldsymbol{\theta})+\sigma^{2}(\boldsymbol{\theta}) \mathbf{I}\right\} \text { with } \\
\sigma^{2}(\boldsymbol{\theta})=\frac{1}{N-M} \operatorname{tr}\left\{\mathbf{P}_{\mathbf{A}}^{\perp} \widehat{\mathbf{R}}_{\mathrm{ML}}\right\}, \\
\mathbf{B}(\boldsymbol{\theta})=\left(\mathbf{A}^{H} \mathbf{A}\right)^{-1} \mathbf{A}^{H}\left(\widehat{\mathbf{R}}_{\mathrm{ML}}-\sigma^{2}(\boldsymbol{\theta}) \mathbf{I}\right) \mathbf{A}\left(\mathbf{A}^{H} \mathbf{A}\right)^{-1} \\
\text { for } \mathbf{A}=\mathbf{A}(\boldsymbol{\theta}) .
\end{gathered}
$$

The deterministic ML method has some intuitive interpretations:

(1) $F_{\text {det }}(\boldsymbol{\theta})=(1 / K) \sum_{k=\mathbf{1}}^{K} \mathbf{z}_{k}^{H} \mathbf{P}_{\mathbf{A}}^{\perp} \mathbf{z}_{k}=(1 / K) \sum_{k=1}^{K}\left\|\mathbf{P}_{\mathbf{A}}^{\perp} \mathbf{z}_{k}\right\|^{2}=$ $(1 / K) \sum_{k=1}^{K}\|\mathbf{z}_{k}-\underbrace{\mathbf{A}}_{=\widehat{\mathbf{b}}} \underbrace{\left(\mathbf{A}^{H} \mathbf{A}\right)^{-1} \mathbf{A}^{H} \mathbf{z}_{k}}\|^{2}$, which means that the mean squared residual error after signal extraction is minimized.

(2) $F_{\text {det }}(\boldsymbol{\theta})=C-\sum_{k=1}^{K} \mathbf{z}_{k}^{H} \mathbf{A}\left(\mathbf{A}^{H} \mathbf{A}\right)^{-1} \mathbf{A}^{H} \mathbf{z}_{k}$, which can be interpreted as maximizing a set of decoupled sum beams $\left(\mathbf{a}^{H}\left(\mathbf{u}_{1}\right) \mathbf{z}_{\mathbf{k}}, \ldots, \mathbf{a}^{H}\left(\mathbf{u}_{M}\right) \mathbf{z}_{\mathbf{k}}\right)$.

(3) $F_{\text {det }}(\boldsymbol{\theta})=C-\mathbf{a}_{\text {null }}^{H} \widehat{\mathbf{R}} \mathbf{a}_{\text {null }} / \mathbf{a}_{\text {null }}^{H} \mathbf{a}_{\text {null }}$ with $\mathbf{a}_{\text {null }}=\mathbf{P}_{\breve{\mathbf{A}}}^{\perp} \mathbf{a}(\mathbf{u})$, where we have partitioned the matrix of steering vectors into $\mathbf{A}=(\mathbf{a}, \breve{\mathbf{A}})$. This property is valid due to the projection decomposition lemma which says that for any partitioning $\mathbf{A}=(\mathbf{F}, \mathbf{G})$ we can write $\mathbf{P}_{\mathbf{A}}^{\perp}=$ $\mathbf{P}_{\mathbf{G}}^{\perp}-\mathbf{P}_{\mathbf{G}}^{\perp} \mathbf{F}\left(\mathbf{F}^{H} \mathbf{P}_{\mathbf{G}}^{\perp} \mathbf{F}\right)^{-1} \mathbf{F}^{H} \mathbf{P}_{\mathbf{G}}^{\perp}$. If we keep the directions in $\breve{A}$ fixed, this relation says that we have to maximize the scan pattern over $\mathbf{u}$ while the sources in the directions of $\breve{\mathbf{A}}$ are deterministically nulled (see (7)). One can 


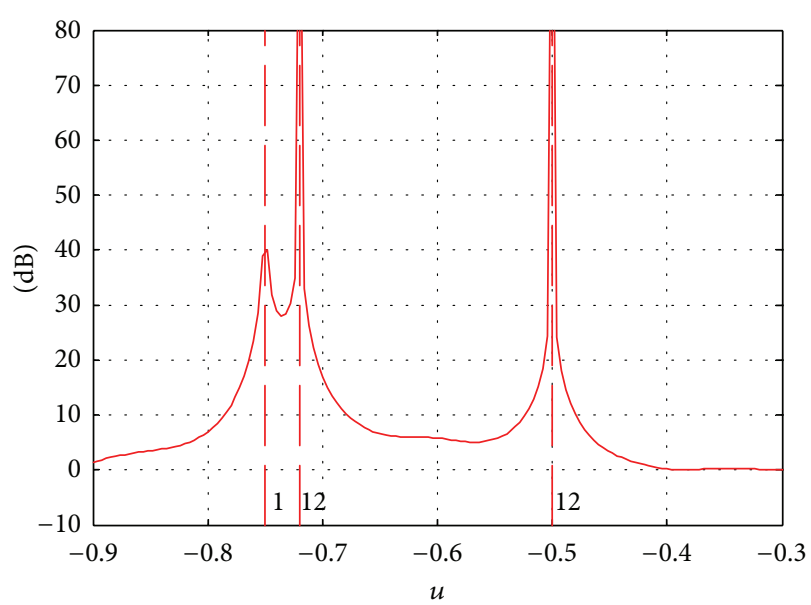

(a) Simulated data

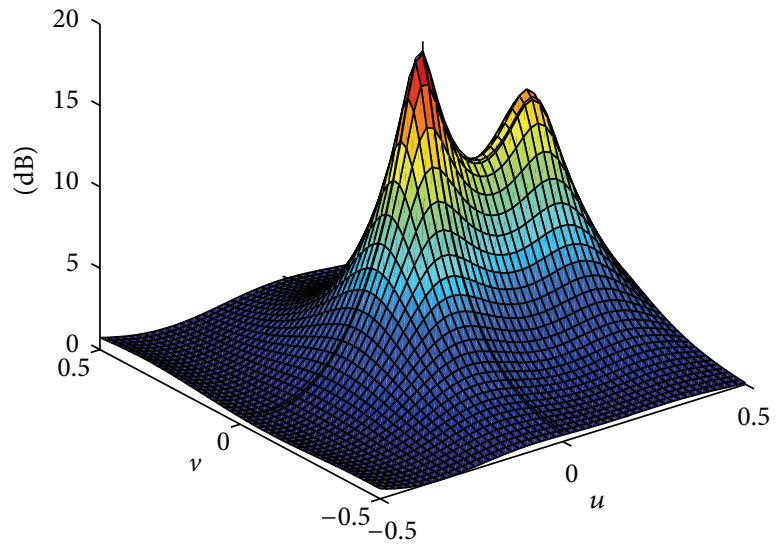

(b) Measured real data

FIGURE 9: MUSIC spectra with a planar array of 7 elements.

now perform the multidimensional maximization by alternating 1-dimensional maximizations and keeping the remaining directions fixed. This is the basis of the alternating projection (AP) method or IMP (Incremental MultiParameter) method, [22, page 105].

A typical feature of the MUSIC method is illustrated in Figure 9. This figure shows the excellent resolution in simulation while for real data the pattern looks almost the same as with Capon's method.

A result with real data with the deterministic ML method is shown in Figure 10. Minimization was performed here with a stochastic approximation method. This example shows in particular that the deterministic ML-method is able to resolve highly correlated targets which arise due to the reflection on the sea surface for low angle tracking. The behavior of the monopulse estimates reflect the variation of the phase differences of direct and reflected path between $0^{\circ}$ and $180^{\circ}$. For $0^{\circ}$ phase difference the monopulse points into the centre, for $180^{\circ}$ it points outside the 2 -target configuration.

The problems of superresolution methods are described in $[21,23]$. A main problem is the numerical effort of finding the $M$ maxima (one $M$-dimensional optimization or $M 1$ dimensional optimizations for a linear antenna). To mitigate this problem a stochastic approximation algorithm or the IMP method has been proposed for the deterministic ML method. The IMP method is an iteration of maximizations of an adaptively formed beam pattern. Therefore, the generalized monopulse method can be used for this purpose, see Section 7.1 and [24].

Another problem is the exact knowledge of the signal model for all possible directions (the vector function $\mathbf{a}(\mathbf{u})$ ). The codomain of this function is sometimes called the array manifold. This is mainly a problem of antenna accuracy or calibration. While the transmission of a plane wave in the main beam direction can be quite accurately modeled (using calibration) this can be difficult in the sidelobe region.

For an array with digital subarrays, superresolution has to be performed only with these subarray outputs. The array manifold has then to be taken at the subarray outputs as in (2). This manifold (the subarray patterns) is well modeled in the main beam region but often too imprecise in the sidelobe region to obtain a resolution better than the conventional. In that case it is advantageous to use a simplified array manifold model based only on the subarray gains and centers, called the Direct Uniform Manifold model (DUM). This simplified model has been successfully applied to MUSIC (called Spotlight MUSIC, [25]) and to the deterministic ML method. Using the DUM model requires little calibration effort and gives improved performance, [25].

More refined parametric methods with higher asymptotic resolution property have been suggested (e.g., COMET, Covariance Matching Estimation Technique, [26]). However, application of such methods to real data often revealed no improvement (as is the case with MUSIC in Figure 9). The reason is that these methods are much more sensitive to the signal model than the accuracy of the system provides. A sensitivity with an very sharp ideal minimum of the objective function may lead to a measured data objective function where the minimum has completely disappeared.

5.2. Target Number Determination. Superresolution is a combined target number and target parameter estimation problem. As a starting point all the methods of Section 4.3 can be used. If we use the detML method we can exploit that the objective function can be interpreted as the residual error between model (interpretation 2) and data. The WNT test statistic (23) is just an estimate of this quantity. The detML residual can therefore be used for this test instead of the sum of the eigenvalues.

These methods may lead to a possibly overestimated target number. To determine the power allocated to each target a refined ML power estimate using the estimated directions $\mathbf{A}(\boldsymbol{\theta})$ can be used $\mathbf{B}(\boldsymbol{\theta})=\left(\mathbf{A}^{H} \mathbf{A}\right)^{-1} \mathbf{A}^{H}\left(\widehat{\mathbf{R}}_{\mathrm{ML}}-\right.$ $\left.\sigma^{2}(\boldsymbol{\theta}) \mathbf{I}\right) \mathbf{A}\left(\mathbf{A}^{H} \mathbf{A}\right)^{-1}$ with $\sigma^{2}(\boldsymbol{\theta})=(1 /(N-M)) \operatorname{tr}\left\{\mathbf{P}_{\mathbf{A}}^{\perp} \widehat{\mathbf{R}}_{\mathrm{ML}}\right\}$ as in (30). This estimate can even reveal correlations between the targets. This has been successfully demonstrated with 


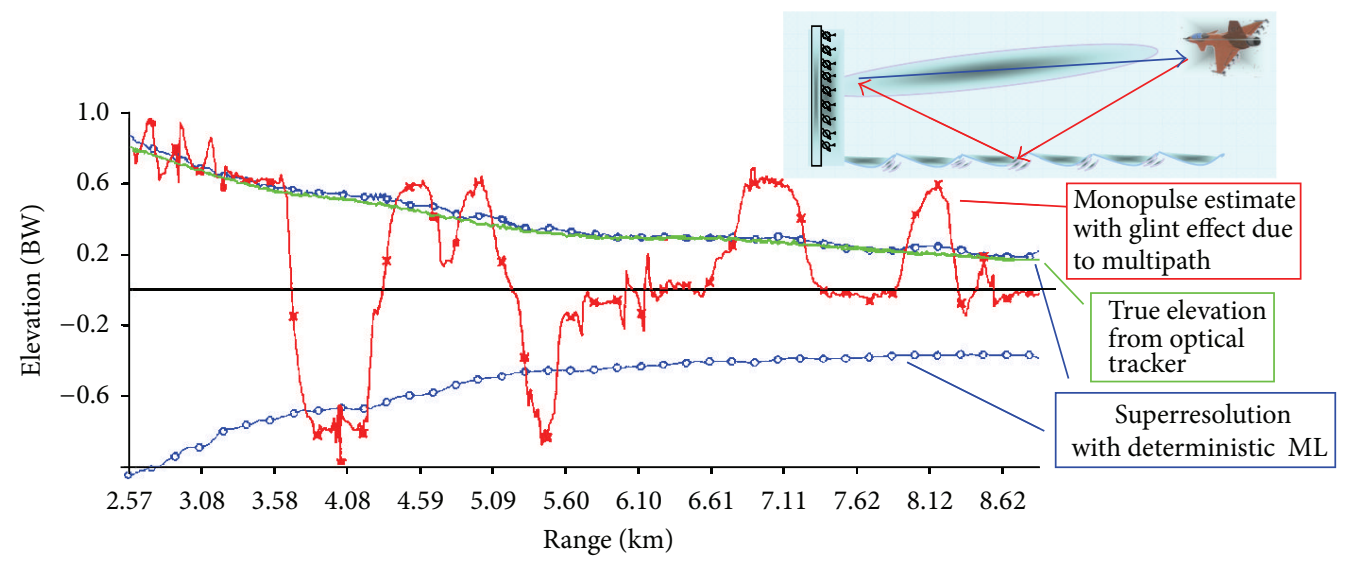

FIGURE 10: Superresolution of multipath propagation over sea with deterministic ML method (real data from vertical linear array with 32 elements, scenario illustrated above).

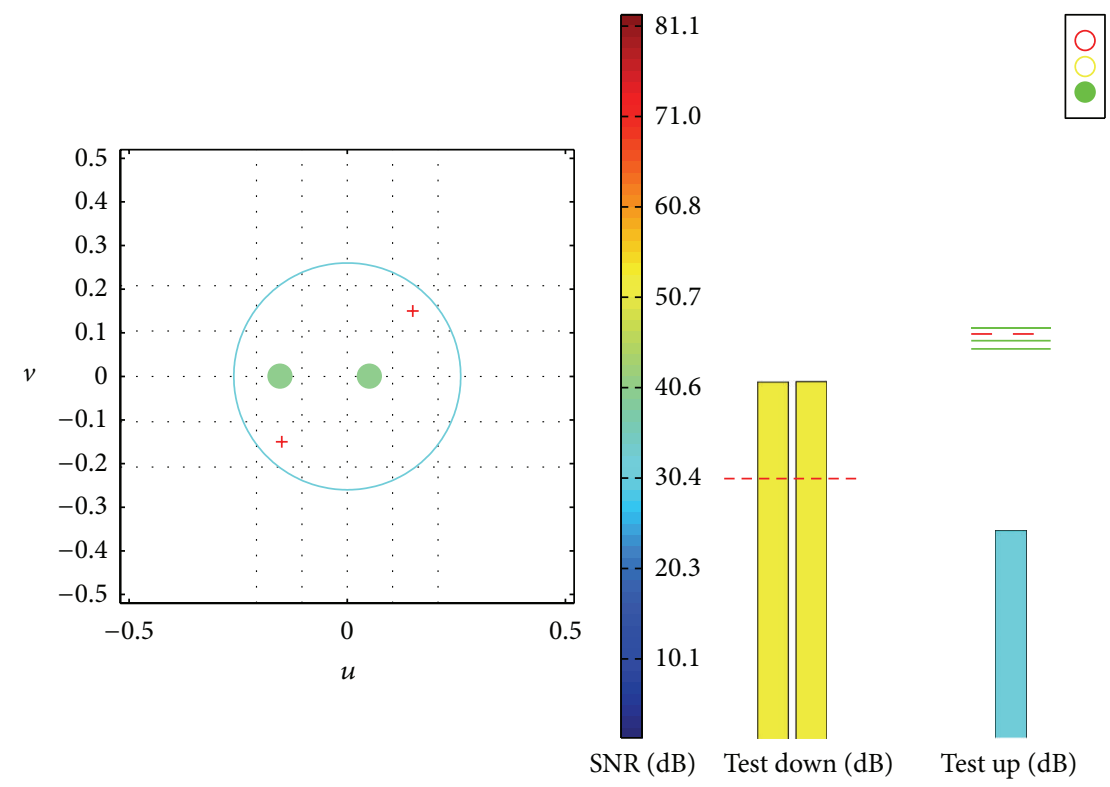

FIGURE 11: Combined target number and direction estimation for 2 targets with 7-element planar array.

the low angle tracking data of Figure 10. In case that some target power is too low, the target number can be reduced and the angle estimates can be updated. This is an iterative procedure of target number estimation and confirmation or reduction. This way, all target modeling can be accurately matched to the data.

The deterministic ML method (28) together with the white noise test (24) is particularly suited for this kind of iterative model fitting. It has been implemented in an experimental system with a 7-element planar array at Fraunhofer FHR and was first reported in [21, page 81]. An example of the resulting output plot is shown in Figure 11. The estimated directions in the $u, v$-plane are shown by small dishes having a color according to the estimated target SNR corresponding to the color bar. The circle indicates the $3 \mathrm{~dB}$ contour of the sum beam. One can see that the two targets are at about 0.5 beamwidth separation. The directions were estimated by the stochastic approximation algorithm used in Figure 10. The test statistic for increasing the target number is shown by the right most bar. The thresholds for increasing the number are indicated by lines. The dashed line is the actually valid threshold (shown is the threshold for 2 targets). The target number can be reduced if the power falls below a threshold shown in two yellow bars in the middle. The whole estimation and testing procedure can also be performed adaptively with changing target situations. We applied it to two blinking targets alternating between the states "target 1 on", "both targets on", "target 2 on", "both targets on", and so forth. Clearly, these test works only if the estimation procedure has converged. This is indicated by the traffic light in the right up corner. We used a fixed empirically determined iteration number to switch the test procedure on (=green traffic light). 


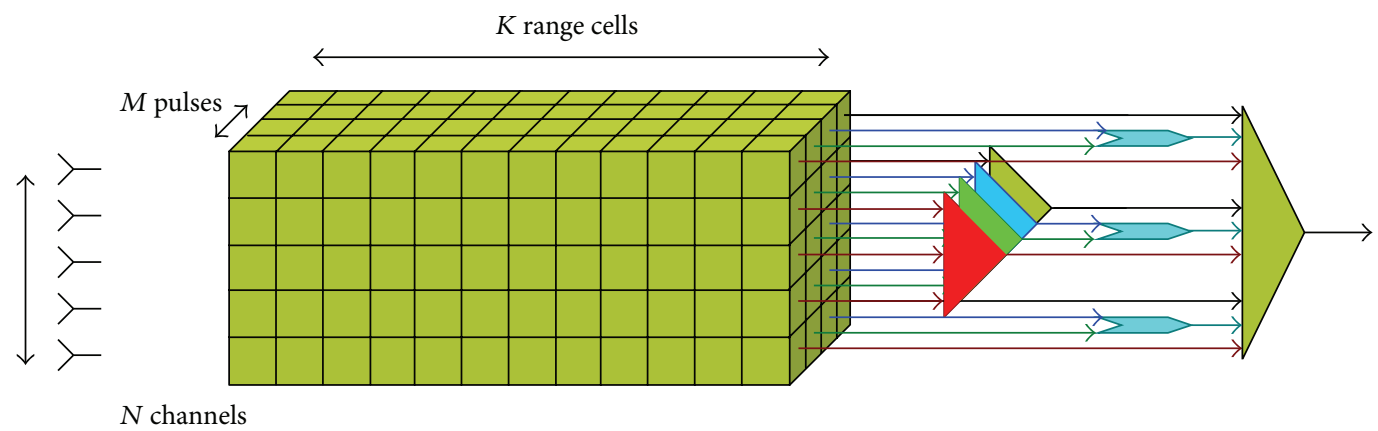

FIGURE 12: Symmetric auxiliary sensor/echo processor of Klemm [4], as an example for forming space-time subarrays.

All thresholds and iteration numbers have to be selected carefully. Otherwise, situations may arise where this adaptive procedure switches between two target models, for example, between 2 and 3 targets.

The problem of resolution of two closely spaced targets becomes a particular problem in the so called threshold region, which denotes configurations where the SNR or the separation of the targets lead to an angular variance departing significantly from the Cramer-Rao bound (CRB). The design of the tests and this threshold region must be compatible to give consistent joint estimation-detection resolution result. These problems have been studied in $[27,28]$. One way to achieve consistency and improving resolution proposed in [27] is to detect and remove outliers in the data, which are basically responsible for the threshold effect. A general discussion about the achievable resolution and the best realistic representation of a target cluster can be found in [28].

\section{Extension to Space-Time Arrays}

As mentioned in Section 2.4, there is mathematically no difference between spatial and temporal samples as long as the distributional assumptions are the same. The adaptive methods and superresolution methods presented in the previous sections can therefore be applied analogously in the time or space-time domain.

In particular, subarraying in time domain is an important tool to reduce the numerical complexity for space-time adaptive processing (STAP) which is the general approach for adaptive clutter suppression for airborne radar, [4]. With the formalism of transforming space-time 2D-beamforming of a data matrix into a usual beamforming operation of vectors introduced in (4), the presented adaptive beamforming and superresolution methods can be easily transformed into corresponding subarrayed space-time methods.

Figure 12 shows an example of an efficient space-time subarraying scheme used for STAP clutter cancellation for airborne radar.

\section{Embedding of Array Processing into Full Radar Data Processing}

A key problem that has to be recognized is that the task of a radar is not to maximize the $\mathrm{SNR}$, but to give the best relevant information about the targets after all processing. This means that for implementing refined methods of interference suppression or superresolution we have also to consider the effect on the subsequent processing. To get optimum performance all subsequent processing should exploit the properties of the refined array signal processing methods applied before. In particular it has been shown that for the tasks of detection, angle estimation and tracking significant improvements can be achieved by considering special features.

7.1. Adaptive Monopulse. Monopulse is an established technique for rapid and precise angle estimation with array antennas. It is based on two beams formed in parallel, a sum beam and a difference beam. The difference beam is zero at the position of the maximum of the sum beam. The ratio of both beams gives an error value that indicates the offset of a target from the sum beam pointing direction. In fact, it can be shown that this monopulse estimator is an approximation of the Maximum-Likelihood angle estimator, [24]. The monopulse estimator has been generalized in [24] to arrays with arbitrary subarrays and arbitrary sum and difference beams.

When adaptive beams are used the shape of the sum beam will be distorted due to the interference that is to be suppressed. The difference beam must adaptively suppress the interference as well, which leads to another distortion. Then the ratio of both beams will no more indicate the target direction. The generalized monopulse procedure of [24] provides correction values to compensate these distortions.

The generalized monopulse formula for estimating angles $(\widehat{u}, \widehat{v})^{T}$ with a planar array and sum and difference beams formed into direction $\left(u_{0}, v_{0}\right)^{T}$ is

$$
\left(\begin{array}{l}
\widehat{u} \\
\widehat{v}
\end{array}\right)=\left(\begin{array}{l}
u_{0} \\
v_{0}
\end{array}\right)-\left(\begin{array}{ll}
c_{x x} & c_{x y} \\
c_{y x} & c_{y y}
\end{array}\right)\left(\begin{array}{l}
R_{x}-\mu_{x} \\
R_{y}-\mu_{y}
\end{array}\right),
$$

where $\mathbf{C}=\left(\begin{array}{ll}c_{x x} & c_{x y} \\ c_{y x} & c_{y y}\end{array}\right)$ is a slope correction matrix and $\boldsymbol{\mu}=\left(\begin{array}{l}\mu_{x} \\ \mu_{y}\end{array}\right)$ is a bias correction. $R_{x}=\operatorname{Re}\left\{\mathbf{d}_{x}^{H} \mathbf{z} / \mathbf{w}^{H} \mathbf{z}\right\}$ is the monopulse ratio formed with the measured difference and sum beam outputs $D_{x}=\mathbf{d}_{x}^{H} \mathbf{z}$ and $S=\mathbf{w}^{H} \mathbf{z}$, respectively, with difference and sum beam weight vectors $\mathbf{d}_{x}, \mathbf{w}$ (analogous for elevation estimation with $\mathbf{d}_{y}$ ). The monopulse ratio is a function of the unknown target directions $(u, v)$. Let the vector of monopulse ratios be denoted by $\mathbf{R}(u, v)=\left(R_{x}(u, v), R_{y}(u, v)\right)^{T}$. The correction quantities are determined such that the expectation 
of the error is unbiased and a linear function with slope 1 is approximated. More precisely, for the following function of the unknown target direction:

$$
\mathbf{M}(u, v)=\mathbf{C} \cdot(E\{\mathbf{R}(u, v)\}-\boldsymbol{\mu}),
$$

we require

$$
\begin{gathered}
\mathbf{M}\left(u_{0}, v_{0}\right)=\mathbf{0}, \\
\frac{\partial \mathbf{M}}{\partial u}\left(u_{0}, v_{0}\right)=\left(\begin{array}{l}
1 \\
0
\end{array}\right), \quad \frac{\partial \mathbf{M}}{\partial v}\left(u_{0}, v_{0}\right)=\left(\begin{array}{l}
0 \\
1
\end{array}\right) \text { or } \\
\mathbf{C}\left(\frac{\partial \mathbf{R}}{\partial u} \frac{\partial \mathbf{R}}{\partial v}\right)\left(u_{0}, v_{0}\right)=\mathbf{I} .
\end{gathered}
$$

These conditions can only approximately be fulfilled for sufficiently high SNR. Then, one obtains for the bias correction for a pointing direction $\mathbf{a}_{0}=\mathbf{a}\left(u_{0}, v_{0}\right)$, [24]

$$
\mu_{\alpha}=\operatorname{Re}\left\{\frac{\mathbf{d}_{\alpha}^{H} \mathbf{a}_{0}}{\mathbf{w}^{H} \mathbf{a}_{0}}\right\} \quad \text { for } \alpha=x, y .
$$

For the elements of the inverse slope correction matrix

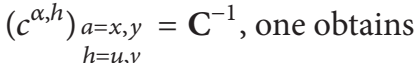

$$
c^{\alpha, h}=\frac{\operatorname{Re}\left\{\mathbf{d}_{\alpha}^{H} \mathbf{a}_{h, 0} \mathbf{a}_{0}^{H} \mathbf{w}+\mathbf{d}_{\alpha}^{H} \mathbf{a}_{0} \mathbf{a}_{h, 0}^{H} \mathbf{w}\right\}}{\left|\mathbf{w}^{H} \mathbf{a}_{0}\right|^{2}}-\mu_{a} 2 \operatorname{Re}\left\{\frac{\mathbf{w}^{H} \mathbf{a}_{h, 0}}{\mathbf{w}^{H} \mathbf{a}_{0}}\right\}
$$

with $\alpha=x$ or $y$ and $h=u$ or $v$, and $\mathbf{a}_{h, 0}$ denotes the derivative $\left.(\partial \mathbf{a} / \partial h)\right|_{\left(u_{0}, v_{0}\right)}$. In general, these are fixed antenna determined quantities. For example, for omnidirectional antenna elements, and phase steering at the elements we have $\mathbf{a}_{0}=G_{e}(1, \ldots, 1)^{T}$, where $G_{e}$ is the antenna element gain, and $\mathbf{a}_{u, 0}^{T}=G_{e}(j 2 \pi f / c)\left(x_{1}, \ldots, x_{N}\right)$.

It is important to note that this formula is independent of any scaling of the difference and sum weights. Constant factors in the difference and sum weight will be cancelled by the corresponding slope correction. Figure 13 shows theoretically calculated bias and variances for this corrected generalized monopulse using the formulas of [24] for the array of Figure 2. The biases are shown by arrows for different possible single target positions with the standard deviation ellipses at the tip. A jammer is located in the asterisk symbol direction with JNR $=27 \mathrm{~dB}$. The hypothetical target has a SNR of $6 \mathrm{~dB}$. The $3 \mathrm{~dB}$ contour of the unadapted sum beam is shown by a dashed circle. The $3 \mathrm{~dB}$ contour of the adapted beam will be of course different. One can see that in the beam pointing direction $(0,0)$ the bias is zero and the variance is small. The errors increase for target directions on the skirt of the main beam and close to the jammer.

The large bias may not be satisfying. However, one may repeat the monopulse procedure by repeating the monopulse estimate with a look direction steered at subarray level into the new estimated direction. This is an all-offline procedure with the given subarray data. No new transmit pulse is needed. We have called this the multistep monopulse procedure [24]. Multistep monopulse reduces the bias considerably

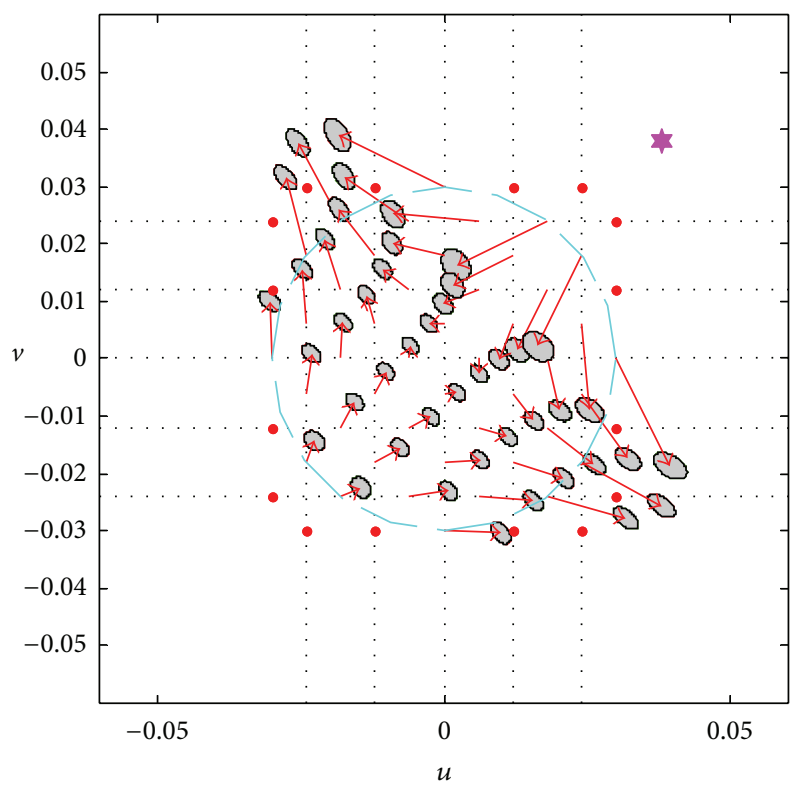

FIGURE 13: Bias and standard deviation ellipses for different target positions.

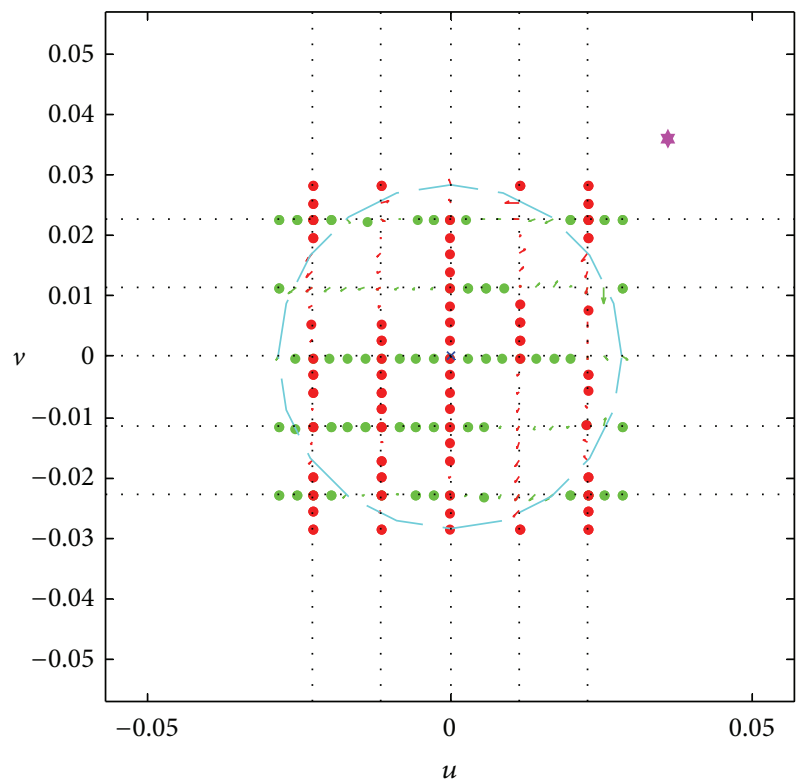

FIGURE 14: Bias for 2-step monopulse for different target positions and jammer scenario of Figure 13.

with only one additional iteration as shown in Figure 14. The variances appearing in Figure 13 are virtually not changed with the multistep monopulse procedure and are omitted for better visibility.

7.2. Adaptive Detection. For detection with adaptive beams, the normal test procedure is not adequate because we have a test statistic depending on two different kinds of random data: the training data for the adaptive weight and the data under test. Various kinds of tests have been developed accounting 
for this fact. The first and basic test statistics were the GLRT, [29], the AMF detector, [30], and the ACE detector, [31]. These have the form

$$
\begin{gathered}
T_{\mathrm{GLRT}}(\widetilde{\mathbf{z}})=\frac{\left|\widetilde{\mathbf{a}}_{0}^{H} \widehat{\mathbf{Q}}_{\mathrm{SMI}}^{-1} \widetilde{\mathbf{z}}\right|^{2}}{\widetilde{\mathbf{a}}_{0}^{H} \widehat{\mathbf{Q}}_{\mathrm{SMI}}^{-1} \widetilde{\mathbf{a}}_{0}\left(1+(1 / K) \widetilde{\mathbf{z}}^{H} \widehat{\mathbf{Q}}_{\mathrm{SMI}}^{-1} \widetilde{\mathbf{z}}\right)}, \\
T_{\mathrm{AMF}}(\widetilde{\mathbf{z}})=\frac{\left|\widetilde{\mathbf{a}}_{0}^{H} \widehat{\mathbf{Q}}_{\mathrm{SMI}}^{-1} \widetilde{\mathbf{z}}\right|^{2}}{\widetilde{\mathbf{a}}_{0}^{H} \widehat{\mathbf{Q}}_{\mathrm{SMI}}^{-1} \widetilde{\mathbf{a}}_{0}}, \\
T_{\mathrm{ACE}}(\widetilde{\mathbf{z}})=\frac{\left|\widetilde{\mathbf{a}}_{0}^{H} \widehat{\mathbf{Q}}_{\mathrm{SMI}}^{-1} \widetilde{\mathbf{z}}\right|^{2}}{\widetilde{\mathbf{a}}_{0}^{H} \widehat{\mathbf{Q}}_{\mathrm{SMI}}^{-1} \widetilde{\mathbf{a}}_{0} \cdot \widetilde{\mathbf{z}}^{H} \widehat{\mathbf{Q}}_{\mathrm{SMI}}^{-1} \widetilde{\mathbf{z}}} .
\end{gathered}
$$

The quantities $\widetilde{\mathbf{z}}, \widetilde{\mathbf{a}}_{0}, \widehat{\mathbf{Q}}$ are here all generated at the subarray outputs, $\widetilde{\mathbf{a}}_{0}$ denotes the plane wave model for a direction $\mathbf{u}_{0}$.

Basic properties of these tests are

$$
\text { (i) } \quad T_{\mathrm{GLRT}}=\frac{T_{\mathrm{AMF}}}{1+(1 / K) \widetilde{\mathbf{z}}^{H} \widehat{\mathbf{Q}}_{\mathrm{SMI}}^{-1} \widetilde{\mathbf{z}}}, \quad T_{\mathrm{ACE}}=\frac{T_{\mathrm{AMF}}}{\widetilde{\mathbf{z}}^{H} \widehat{\mathbf{Q}}_{\mathrm{SMI}}^{-1} \widetilde{\mathbf{z}}} .
$$

(ii) The AMF detector represents an estimate of the signal-tonoise ratio because it can be written as

$$
T_{\mathrm{AMF}}=\frac{\left|\widetilde{\mathbf{w}}^{H} \widetilde{\mathbf{z}}\right|^{2}}{\widetilde{\mathbf{w}}^{H} \widehat{\mathbf{Q}}_{\mathrm{SMI}} \widetilde{\mathbf{w}}} \text { with } \widetilde{\mathbf{w}}=\widehat{\mathbf{Q}}_{\mathrm{SMI}}^{-1} \widetilde{\mathbf{a}}_{0} .
$$

This provides a meaningful physical interpretation. A complete statistical description of these tests has been given in very compact form in $[32,33]$. These results are valid as well for planar arrays with irregular subarrays and also mismatched weighting vector.

Actually, all these detectors use the adaptive weight of the SMI algorithm which has unsatisfactory performance as mentioned in Section 4.2. The unsatisfactory finite sample performance is just the motivation for introducing weight estimators like LSMI, LMI, or CAPS. Clutter, insufficient adaptive suppression and surprise interference are the motivation for requiring low sidelobes. Recently several more complicated adaptive detectors have been introduced with the aim of achieving additional robustness properties, [3438]. However, another and quite simple way would be to generalize the tests of (36), (37), (38) to arbitrary weight vectors with the aim of inserting well known robust weights as derived in Section 4.1. This has been done in [39]. First, we observe that the formulation of (40) can be used for any weight vector. Second, one can observe that the ACE and GLRT have the form of a sidelobe blanking device. In particular it has already been shown in [35] that diagonal loading provides significant better detection performance.

A guard channel is implemented in radar systems to eliminate impulsive interference (hostile or from other neighboring radars) using the sidelobe blanking (SLB) device. The guard channel receives data from an omnidirectional antenna element which is amplified such that its power level is above the sidelobe level of the highly directional radar antenna, but below the power of the radar main beam, [1, page 9.9]. If the received signal power in the guard channel is above the power of the main channel, this must be a signal coming via the sidelobes. Such signals will be blanked. If the guard channel power is below the main channel power it is considered as a detection.

With phased arrays it is not necessary to provide an external omnidirectional guard channel. Such a channel can be generated from the antenna itself; all the required information is in the antenna. We may use the noncoherent sum of the subarrays as guard channel. This is the same as the average omnidirectional power. Some shaping of the guard pattern can be achieved by using a weighting for the noncoherent sum:

$$
G=\sum_{i=1}^{L} \widetilde{g}_{i}\left|\widetilde{z}_{i}\right|^{2}
$$

If all subarrays are equal, a uniform weighting $\widetilde{\mathbf{g}}=(1, \ldots, 1)^{T}$ may be suitable; for unequal irregular subarrays as in Figure 2 the different contributions of the subarrays can be weighted. The directivity pattern of such guard channel is given by $S_{G}(\mathbf{u})=\sum_{i=1}^{L} \widetilde{g}_{i}\left|\widetilde{a}_{i}(\mathbf{u})\right|^{2}$. More generally, we may use a combination of noncoherent and coherent sums of the subarrays with weights contained in the matrices $\mathbf{D}, \mathbf{K}$, respectively,

$$
G=\widetilde{\mathbf{z}}^{H} \mathbf{K D K}^{H_{\widetilde{\mathbf{z}}},} \quad S_{G}(\mathbf{u})=\widetilde{\mathbf{a}}^{H}(\mathbf{u}) \mathbf{K D K}^{H} \widetilde{\mathbf{a}}(\mathbf{u}) .
$$

Examples of such kind of guard channels are shown in Figure 15 for the generic array of Figure 2 with $-35 \mathrm{~dB}$ Taylor weighing for low sidelobes. The nice feature of these guard channels is (i) that they automatically scan together with the antenna look direction, and (ii) that they can easily be made adaptive. This is required if we want to use the SLB device in the presence of CW plus impulsive interference. A CW jammer would make the SLB blank all range cells, that is, would just switch off the radar. To generate an adaptive guard channel we only have to replace in (42) the data vector of the cell under test (CUT) by the pre-whitened data $\widetilde{\mathbf{z}}_{\text {pre- } w}=\widehat{\mathbf{R}}^{-1 / 2} \widetilde{\mathbf{z}}$. Then, the test statistic can be written as $T=T_{\text {AMF }}(\widetilde{\mathbf{z}}) / G_{\text {adapt }}(\widetilde{\mathbf{z}})$, where $G_{\text {adapt }}(\widetilde{\mathbf{z}})=\widetilde{\mathbf{z}}^{H} \widehat{\mathbf{R}}^{-1} \widetilde{\mathbf{z}}$ for ACE and $G_{\text {adapt }}(\widetilde{\mathbf{z}})=1+(1 / K) \widetilde{\mathbf{z}}^{H} \widehat{\mathbf{R}}^{-1} \widetilde{\mathbf{z}}$ for GLRT. Hence $G_{\text {adapt }}$ is just the incoherent sum of the pre-whitened subarray outputs; in other words, $T_{\mathrm{ACE}}$ can be interpreted as an AMF detector with an adaptive guard channel and $T_{\text {GLRT }}$ the same with guard channel on a pedestal. Figure 16 shows examples of some adapted guard channels generated with the generic array of Figure 2 and $-35 \mathrm{~dB}$ Taylor weighting. The unadapted patterns are shown by dashed lines.

This is the adaptive generalization of the usual sidelobe blanking device (SLB) and the AMF, ACE and GLRT tests can be used as extension of the SLB detector to the adaptive case, [32], called the 2D adaptive sidelobe blanking (ASB) detector. The AMF is then the test for the presence of a potential target and the generalized ACE or GRLT are used confirming this target or adaptive sidelobe blanking.

A problem with these modified tests is to define a suitable threshold for detection. For arbitrary weight vector it is nearly impossible to determine this analytically. In [39] the detection 


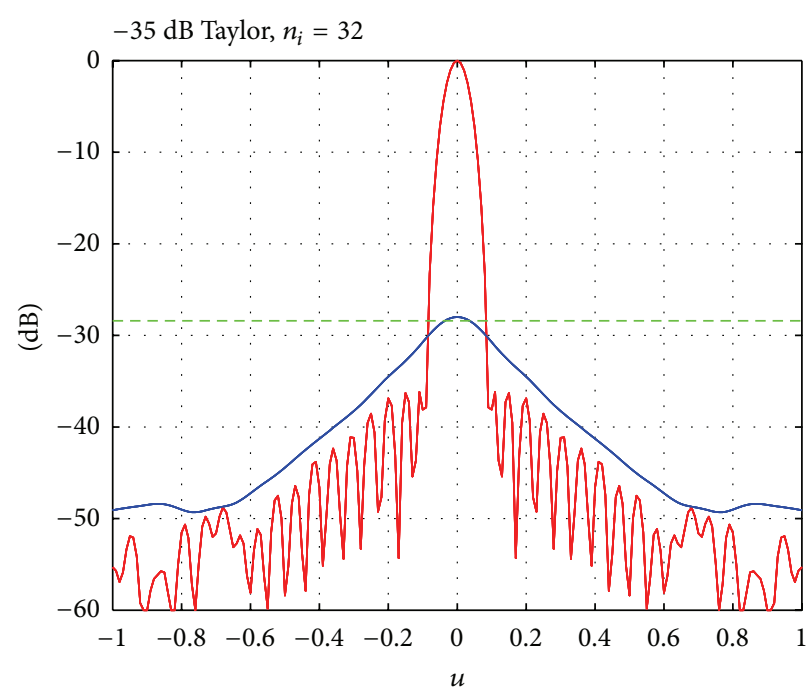

(a) Uniform subarray weighting

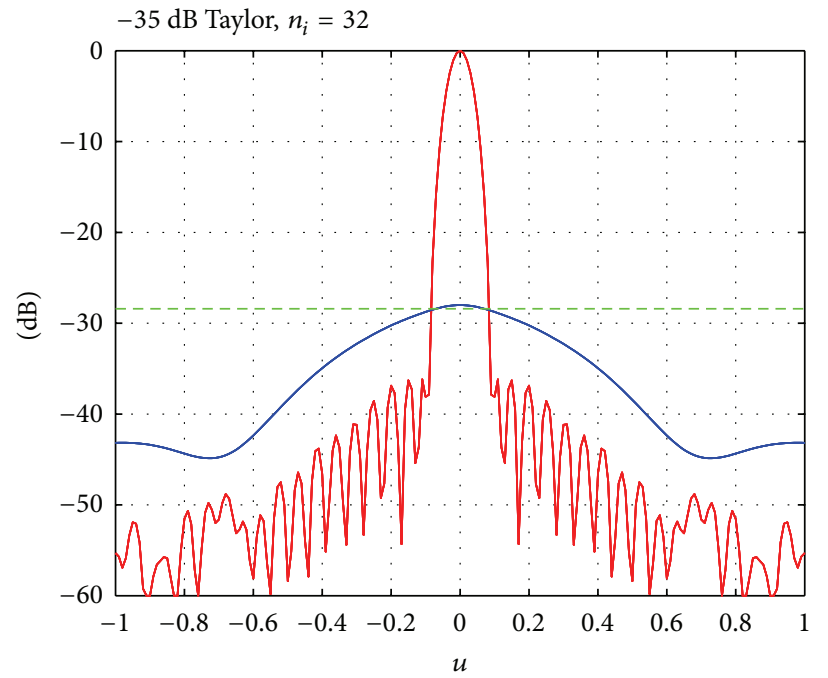

(b) Power equalized weighting

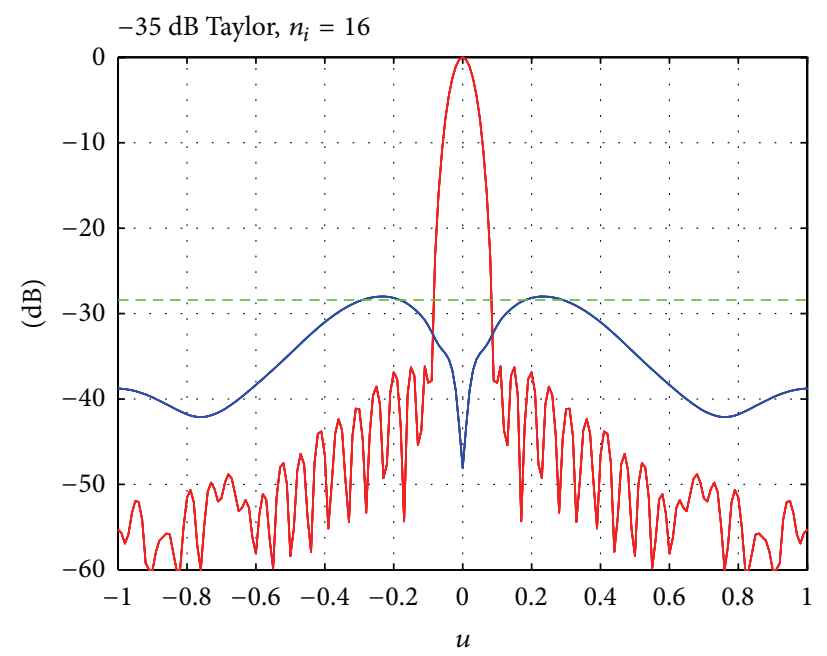

(c) Power equalized + difference weighting

FIGURE 15: Guard channel and sum beam patterns for generic array of Figure 2.

margin has been introduced as an empirical tool for judging a good balance between the AMF and ASB threshold for given jammer scenarios. The detection margin is defined as the difference between the expectation of the AMF statistic and the guard channel, where the expectation is taken only over the interference complex amplitudes for a known interference scenario. In addition one can also calculate the standard deviation of these patterns. The performance against jammers close to the main lobe is the critical feature. The detection margin provides the mean levels together with standard deviations of the patterns. An example of the detection margin is shown in Figure 17 (same antenna and weighting as in Figures 15 and 16).

Comparing the variances of the ACE and GLRT guard channels in [39] revealed that the GLRT guard performs significantly better in terms of fluctuations. The GLRT guard channel may therefore be preferred for its better sidelobe performance and higher statistical stability.
7.3. Adaptive Tracking. A key feature of ABF is that overall performance is dramatically influenced by the proximity of the main beam to an interference source. The task of target tracking in the proximity of a jammer is of high operational relevance. In fact, the information on the jammer direction can be made available by a jammer mapping mode, which determines the direction of the interferences by a background procedure using already available data. Jammers are typically strong emitters and thus easy to detect. In particular, the Spotlight MUSIC method [25] working with subarray outputs is suited for jammer mapping with a multifunction radar.

Let us assume here for simplicity that the jammer direction is known. This is highly important information for the tracking algorithm of a multifunction radar where the tracker determines the pointing direction of the beam. We will use for angle estimation the adaptive monopulse procedure of Section 7.1. ABF will form beams with a notch in the jammer direction. Therefore one cannot expect target echoes from 


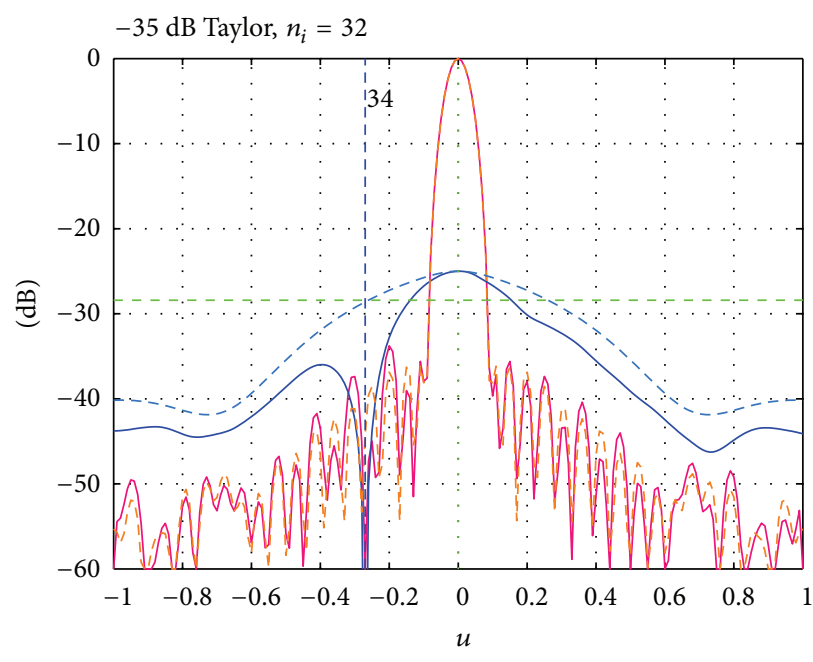

(a) Weighting for equal subarray power

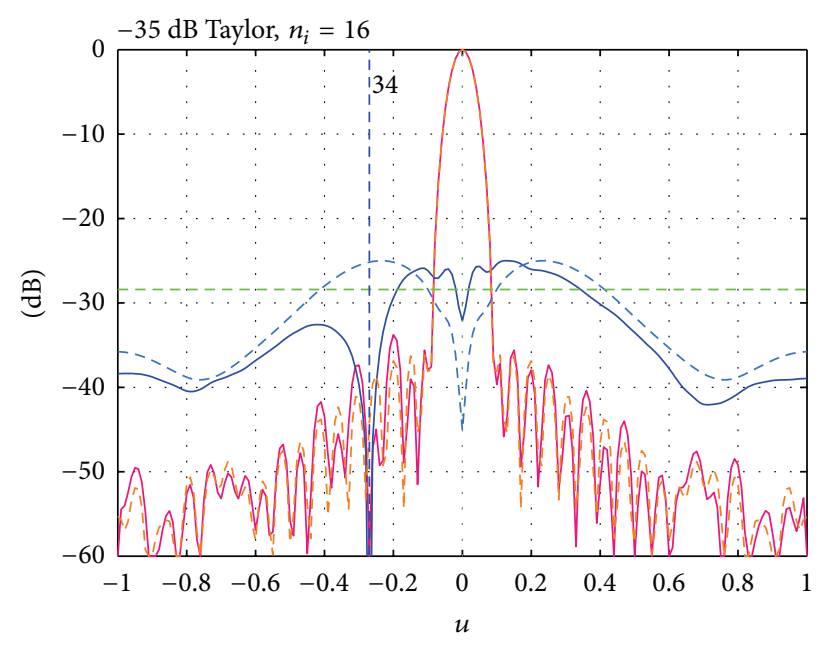

(b) Difference type guard with weighting for equal subarray power

FIGURE 16: Adapted guard patterns for jammer at $u=-0.27\left(-15.7^{\circ}\right)$ with JNR of $34 \mathrm{~dB}$ for generic array.

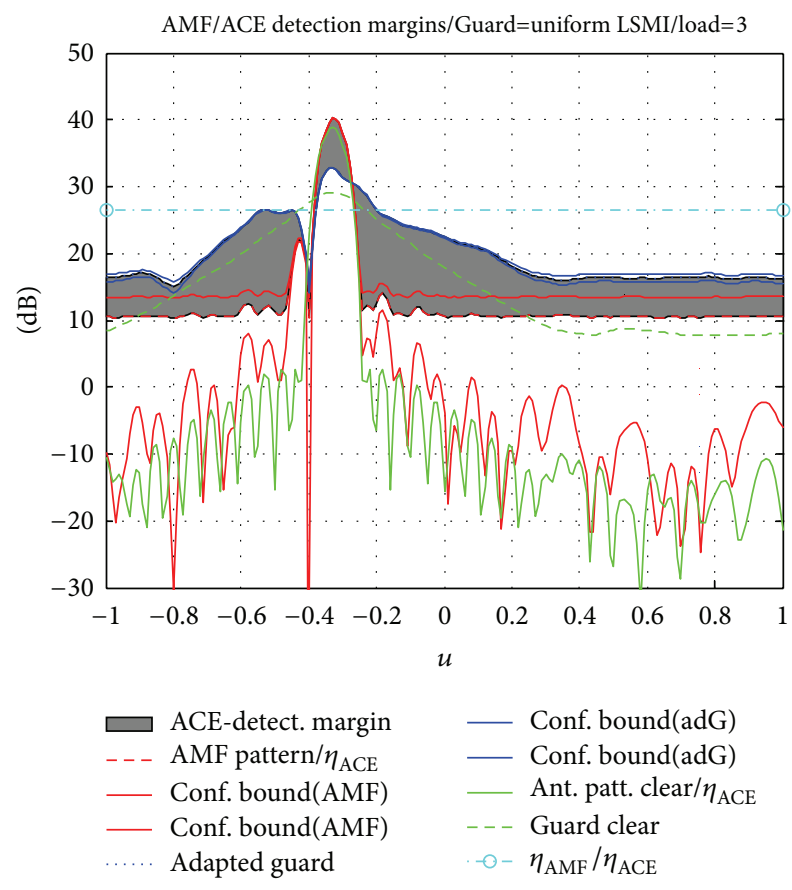

FIGURE 17: Detection margin (gray shading) between AMF and nonweighted adapted guard pattern with confidence bounds for ACE with LSMI (64 snapshots and $3 \sigma^{2}$ diagonal loading). Two CW interferences of $40 \mathrm{~dB}$ INR are present at $u=-0.8,-0.4$.

directions close to the jammer and therefore it does not make sense to steer the beam into the jammer notch. Furthermore, in the case of a missing measurement of a tracked target inside the jammer notch, the lack of a successful detection supports the conclusion that this negative contact is a direct result of jammer nulling by ABF. This is so-called negative information [40]. In this situation we can use the direction of the jammer as a pseudomeasurement to update and maintain the track file. The width of the jammer notch defines the uncertainty of this pseudo measurement. Moreover, if one knows the jammer direction one can use the theoretically calculated variances for the adaptive monopulse estimate of [24] as a priori information in the tracking filter. The adaptive monopulse can have very eccentric uncertainty ellipses as shown in Figure 13 which is highly relevant for the tracker. The large bias appearing in Figure 13, which is not known by the tracker, can be reduced by applying the multistep monopulse procedure, [24].

All these techniques have been implemented in a tracking algorithm and refined by a number of stabilization measures in [41]. The following special measures for ABF tracking have been implemented and are graphically visualized in Figure 18.

(i) Look direction stabilization: the monopulse estimate may deliver measurements outside of the $3 \mathrm{~dB}$ contour of the sum beam. Such estimates are also heavily biased, especially for look directions close to the jammer, despite the use of the multistep monopulse procedure. Estimates of that kind are therefore corrected by projecting them onto the boundary circle of sum beam contour.

(ii) Detection threshold: only those measurements are considered in the update step of the tracking algorithm whose sum beam power is above a certain detection threshold (typically $13 \mathrm{~dB}$ ). This guarantees useful and valuable monopulse estimates. It is well known that the variance of the monopulse estimate decreases monotonically with this threshold increasing.

(iii) Adjustment of antenna look direction: look directions in the jammer notch should generally be avoided due to the expected lack of good measurements. In case that the proposed look direction lies in the jammer notch, we select an adjusted direction on the skirt of the jammer notch. 


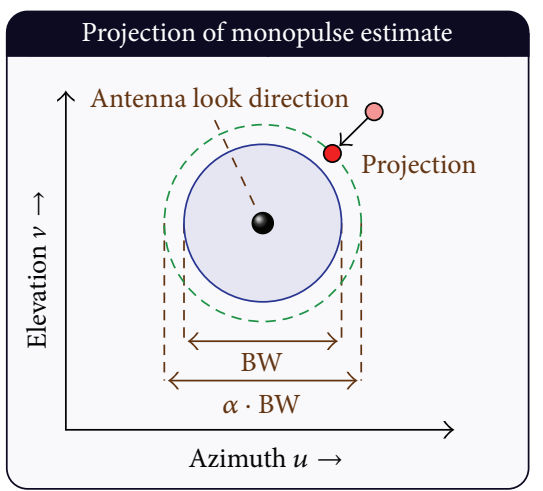

(a)

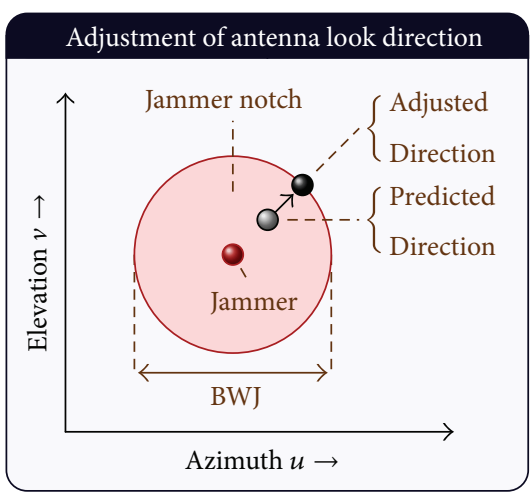

(b)

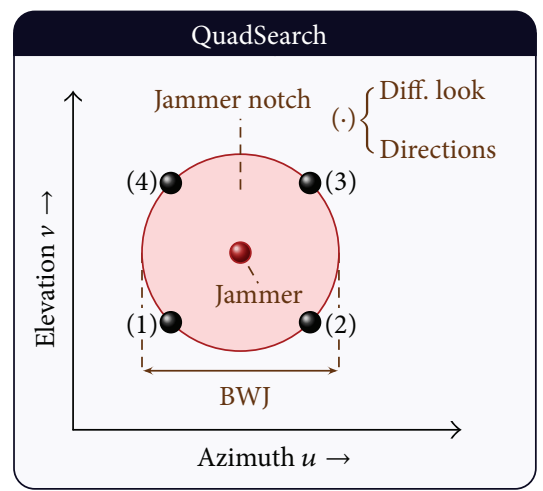

(c)

FIGURE 18: Illustration of different stabilization measures to improve track stability and track continuity.

(iv) Variable measurement covariance: a variable covariance matrix of the adaptive monopulse estimation according to [24] is considered only for a mainlobe jammer situation. For jammers in the sidelobes, there is little effect on the angle estimates, and we can use the fixed covariance matrix of the nonjammed case.

(v) QuadSearch and Pseudomeasurements: if the predicted target direction lies inside the jammer notch and if, despite all adjustments of the antenna look direction, the target is not detected, a specific search pattern is initiated (named QuadSearch) which uses look directions on the skirt of the jammer notch to obtain acceptable monopulse estimates. If this procedure does not lead to a detection, we know that the target is hidden in the jammer notch and we cannot see it. We use then the direction of the jammer as a pseudobearing measurement to maintain the track file. The pseudomeasurement noise is determined by the width of the jammer notch.

(vi) LocSearch: in case of a permanent lack of detections (e.g., for three consecutive scans) while the track position lies outside the jammer notch, a specific search pattern is initiated (named LocSearch) that is similar to the QuadSearch. The new look directions lie on the circle of certain radius around the predicted target direction.

(vii) Modeling of target dynamics: the selection of a suitable dynamics model plays a major role for the quality of tracking results. In this context, the socalled interacting multiple model (IMM) is a wellknown method to reliably track even those objects whose dynamic behavior remains constant only during certain periods.

(viii) Gating: in the vicinity of the jammer, the predicted target direction (as an approximation of the true value) is used to compute the variable angle measurement covariance. Strictly speaking, this is only valid exactly in the particular look direction. Moreover, the tracking algorithm regards all incoming sensor data

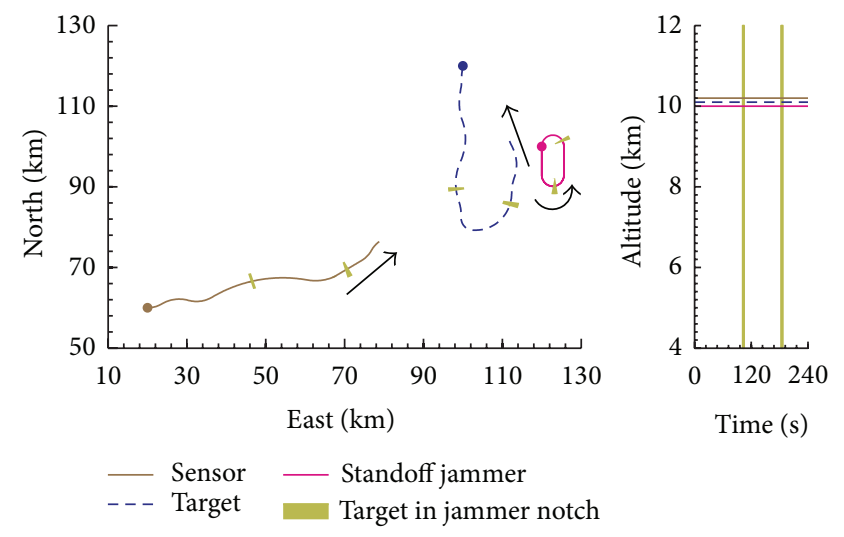

FIGURE 19: Target tracking scenario with standoff jammer: geographic plot of platform trajectories.

as unbiased measurements. To avoid track instabilities, an acceptance region is defined for each measurement depending on the predicted target state and the assumed measurement accuracy. Sensor reports lying outside this gate are considered as invalid.

In order to evaluate our stabilization measures we considered a realistic air-to-air target tracking scenario [41]. Figure 19 provides an overview of the different platform trajectories. In this scenario, the sensor (on a forward looking radar platform flying with a constant speed of $265 \mathrm{~m} / \mathrm{s}$ ) employs the antenna array of Figure 2 (sum beamwidth $\mathrm{BW}=3.4^{\circ}$, field of view $120^{\circ}$, scan interval $1 \mathrm{~s}$ ) and approaches the target (at velocity $300 \mathrm{~m} / \mathrm{s}$ ), which thereupon veers away after a short time. During this time, the target is hidden twice in the jammer notch of the standoff jammer (SOJ) - first for $3 \mathrm{~s}$ and then again for $4 \mathrm{~s}$. The SOJ is on patrol (at $235 \mathrm{~m} / \mathrm{s}$ ) and follows a predefined race track at constant altitude. Figure 20 shows exemplary the evaluation of the azimuth measurements and estimates over time in a window where the target first passes through the jammer notch. The different error bars of a single measurement illustrate the approximation error of the variable measurement covariance: $\sigma_{u_{k}}^{\mathrm{SIM}}$ 

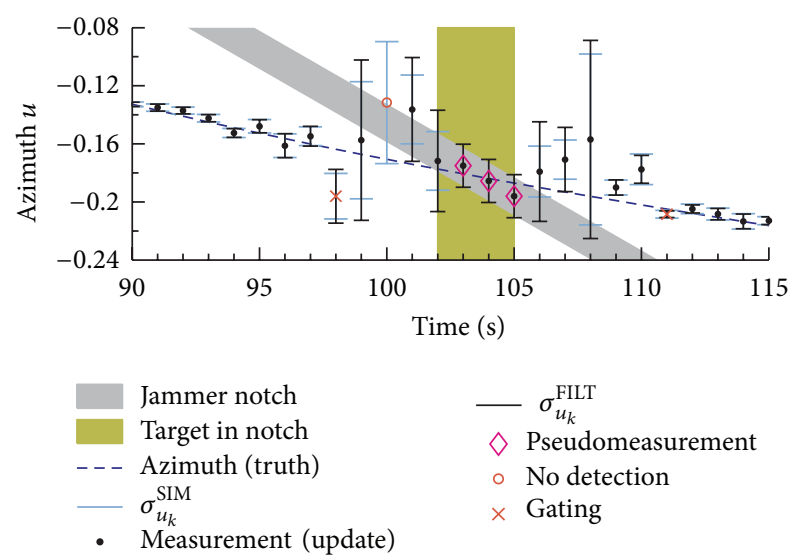

Figure 20: Exemplary azimuth measurements and models for a specific time window for tracking scenario of Figure 19.

denotes the true azimuth standard deviation (std) which is generated in the antenna simulation; $\sigma_{u_{k}}^{\text {FILT }}$ corresponds to the std which is used in the tracking algorithm. More precisely, the tracking program computes the adaptive angle measurement covariance only in the vicinity of the jammer with a diameter of this zone of $8.5^{\circ}$. Outside of this region, the tracking algorithm uses a constant std of 0.004 for both components of the angle measurement. The constant std for the other parameters are $75 \mathrm{~m}$ and $7.5 \mathrm{~m} / \mathrm{s}$ for range and range-rate measurements. The signal-to-noise and jammerto-noise ratios were set to $26 \mathrm{~dB}$ and $27 \mathrm{~dB}$ at a reference range of $70 \mathrm{~km}$. From Figure 20 the benefits of using pseudobearing measurements become apparent.

From these investigations, it turned out that tracking only with adaptive beamforming and adaptive monopulse nearly always leads to track loss in the vicinity of the jammer. With additional stabilization measures that did not require the knowledge of the jammer direction (projection of monopulse estimate, detection threshold, LocSearch, gating) still track instabilities occurred culminating finally in track loss. An advanced tracking version which used pseudomeasurements mitigated this problem to some degree. Finally, the additional consideration of the variable measurement covariance with a better estimate of the highly variable shape of the angle uncertainty ellipse resulted in significantly fewer measurements that were excluded due to gating. In this case all the stabilization measures could not only improve track continuity, but also track accuracy and thus track stability, [41]. This tells us that it is absolutely necessary to use all information of the adaptive process for the tracker to achieve the goal of detection and tracking in the vicinity of the interference.

\section{Conclusions and Final Remarks}

In this paper, we have pointed out the links between array signal processing and antenna design, hardware constraints and target detection, and parameter estimation and tracking. More specifically, we have discussed the following features. (i) Interference suppression by deterministic and adaptive pattern shaping: both approaches can be reasonably combined. Applying ABF after deterministic sidelobe reduction allows reducing the requirements on the low sidelobe level. Special techniques are available to make $\mathrm{ABF}$ preserve the low sidelobe level.

(ii) General principles and relationships between $\mathrm{ABF}$ algorithms and superresolution methods have been discussed, like dependency on the sample number, robustness, the benefits of subspace methods, problems of determining the signal/interference subspace, and interference suppression/resolution limit.

(iii) Array signal processing methods like adaptive beamforming and superresolution methods can be applied to subarrays generated from a large fully filled array. This means applying these methods to the sparse superarray formed by the subarray centers. We have pointed out problems and solutions for this special array problem.

(iv) $\mathrm{ABF}$ can be combined with superresolution in a canonical way by applying the pre-whiten and match principle to the data and the signal model vector.

(v) All array signal processing methods can be extended to space-time processing (arrays) by defining a corresponding space-time plane wave model.

(vi) Superresolution is a joint detection-estimation problem. One has to determine a multitarget model which contains the number, directions and powers of the targets. These parameters are strongly coupled. A practical joint estimation and detection procedure has been presented.

(vii) The problems for implementation in real system have been discussed, in particular the effects of limited knowledge of the array manifold, effect of channel errors, eigenvalue leakage, unequal noise power in array channels, and dynamic range of $\mathrm{AD}$-converters.

(viii) For achieving best performance an adaptation of the processing subsequent to $\mathrm{ABF}$ is necessary. Direction estimation can be accommodated by using ABFmonopulse; the detector can be accommodated by adaptive detection with ASLB, and the tracking algorithms can be extended to adaptive tracking and track management with jammer mapping.

With a single array signal processing method alone no significant improvement will be obtained. The methods have to be reasonably embedded in the whole system, and all functionalities have to be mutually tuned and balanced. This is a task for future research. The presented approaches constitute only a first ad hoc step, and more thorough studies are required. Note that in most cases tuning the functionalities is mainly a software problem. So, there is the possibility to upgrade existing systems softly and step-wise. 


\section{Disclosure}

The main part of this work was performed while the author was with the Fraunhofer Institute for High Frequency Physics and Radar Techniques (FHR) in Wachtberg, Germany.

\section{References}

[1] M. I. Skolnik, Radar Handbook, McGraw Hill, 2nd edition, 1990.

[2] M. A. Richards, J. A. Scheer, and W. A. Holden, Principles of Modern Radar, SciTech Publishing, 2010.

[3] W. D. Wirth, Radar Techniques Using Array Antennas, IEE Publishers, 2001.

[4] R. Klemm, Principles of Space-Time Adaptive Processing, IET Publishers, London, UK, 3rd edition, 2006.

[5] J. H. G. Ender, "On compressive sensing applied to radar," Signal Processing, vol. 90, no. 5, pp. 1402-1414, 2010.

[6] U. Nickel, "Subarray configurations for digital beamforming with low sidelobes and adaptive interference suppression," in Proceedings of the IEEE International Radar Conference, pp. 714719, Alexandria, Egypt, May 1995.

[7] U. Nickel, "Properties of digital beamforming with subarrays," in Proceedings of the International Conference on Radar (CIE '06), pp. 6-19, Shanghai, China, October 2006.

[8] W. Bürger, "Sidelobe forming for ground clutter and jammer suppression for airborne active array radar," in Proceedings of the IEEE International Symposium on Phased Array Systems and Technology, Boston, Mass, USA, 2003.

[9] J. Liu, A. B. Gershman, Z.-Q. Luo, and K. M. Wong, "Adaptive beamforming with sidelobe control: a second-order cone programming approach," IEEE Signal Processing Letters, vol. 10, no. 11, pp. 331-334, 2003.

[10] S. A. Vorobyov, A. B. Gershman, Z.-Q. Luo, and N. Ma, "Adaptive beamforming with joint robustness against mismatched signal steering vector and interference nonstationarity," IEEE Signal Processing Letters, vol. 11, no. 2, pp. 108-111, 2004.

[11] G. A. Fabrizio, A. B. Gershman, and M. D. Turley, "Robust adaptive beamforming for HF surface wave over-the-horizon radar," IEEE Transactions on Aerospace and Electronic Systems, vol. 40, no. 2, pp. 510-525, 2004.

[12] Y. Hua, A. B. Gershman, and Q. Cheng, High Resolution and Robust Signal Processing, Marcel Dekker, 2004.

[13] U. Nickel, "Adaptive Beamforming for Phased Array Radars," in Proceedings of the IEEE International Radar Symposium (IRS '98), pp. 897-906, DGON and VDE/ITG, September 1998.

[14] C. H. Gierull, "Fast and effective method for low-rank interference suppression in presence of channel errors," Electronics Letters, vol. 34, no. 6, pp. 518-520, 1998.

[15] G. M. Herbert, "New projection based algorithm for low sidelobe pattern synthesis in adaptive arrays," in Proceedings of the Radar Edinburgh International Conference, pp. 396-400, October 1997.

[16] U. Nickel, "Determination of the dimension of the signal subspace for small sample size," in Proceedings of the IASTED international conference on Signal Processing and Communication Systems, pp. 119-122, IASTED/Acta Press, 1998.

[17] S. Kritchman and B. Nadler, "Non-parametric detection of the number of signals: hypothesis testing and random matrix theory," IEEE Transactions on Signal Processing, vol. 57, no. 10, pp. 3930-3941, 2009.
[18] U. Nickel, "On the influence of channel errors on array signal processing methods," International Journal of Electronics and Communications, vol. 47, no. 4, pp. 209-219, 1993.

[19] R. J. Muirhead, Aspects of Multivariate Analysis Theory, John Wiley \& Sons, New York, NY, USA, 1982.

[20] R. R. Nadakuditi and A. Edelman, "Sample eigenvalue based detection of high-dimensional signals in white noise using relatively few samples," IEEE Transactions on Signal Processing, vol. 56, no. 7, pp. 2625-2638, 2008.

[21] U. Nickel, "Radar target parameter estimation with antenna arrays," in Radar Array Processing, S. Haykin, J. Litva, and T. J. Shepherd, Eds., pp. 47-98, Springer, 1993.

[22] S. Haykin, Advances in Spectrum Analysis and Array Processing. Vol. II, Prentice Hall, 1991.

[23] U. Nickel, "Aspects of implementing super-resolution methods into phased array radar," International Journal of Electronics and Communications, vol. 53, no. 6, pp. 315-323, 1999.

[24] U. Nickel, "Overview of generalized monopulse estimation," IEEE Aerospace and Electronic Systems Magazine, vol. 21, no. 6, pp. 27-55, 2006.

[25] U. Nickel, "Spotlight MUSIC: super-resolution with subarrays with low calibration effort," IEE Proceedings, vol. 149, no. 4, pp. 166-173, 2002.

[26] B. Ottersten, P. Stoica, and R. Roy, "Covariance matching estimation techniques for array signal processing applications," Digital Signal Processing, vol. 8, no. 3, pp. 185-210, 1998.

[27] Y. I. Abramovich, N. K. Spencer, and A. Y. Gorokhov, "GLRTbased threshold detection-estimation performance improvement and application to uniform circular antenna arrays," IEEE Transactions on Signal Processing, vol. 55, no. 1, pp. 20-31, 2007.

[28] Y. I. Abramovich and B. A. Johnson, "Detection-estimation of very close emitters: performance breakdown, ambiguity, and general statistical analysis of maximum-likelihood estimation," IEEE Transactions on Signal Processing, vol. 58, no. 7, pp. 36473660, 2010.

[29] E. J. Kelly, "Performance of an adaptive detection algorithm; rejection of unwanted signals," IEEE Transactions on Aerospace and Electronic Systems, vol. 25, no. 2, pp. 122-133, 1992.

[30] F. C. Robey, D. R. Fuhrmann, E. J. Kelly, and R. Nitzberg, "A CFAR adaptive matched filter detector," IEEE Transactions on Aerospace and Electronic Systems, vol. 28, no. 1, pp. 208-216, 1992.

[31] S. Kraut and L. L. Scharf, "Adaptive subspace detectors," IEEE Transactions on Signal Processing, vol. 49, no. 1, pp. 1-16, 2001.

[32] C. D. Richmond, "Performance of the adaptive sidelobe blanker detection algorithm in homogeneous environments," IEEE Transactions on Signal Processing, vol. 48, no. 5, pp. 1235-1247, 2000.

[33] C. D. Richmond, "Performance of a class of adaptive detection algorithms in nonhomogeneous environments," IEEE Transactions on Signal Processing, vol. 48, no. 5, pp. 1248-1262, 2000.

[34] T. F. Ayoub and A. M. Haimovich, "Modified GLRT signal detection algorithm," IEEE Transactions on Aerospace and Electronic Systems, vol. 36, no. 3, pp. 810-818, 2000.

[35] Y. I. Abramovich, N. K. Spencer, and A. Y. Gorokhov, "Modified GLRT and AMF framework for adaptive detectors," IEEE Transactions on Aerospace and Electronic Systems, vol. 43, no. 3, pp. 1017-1051, 2007.

[36] O. Besson, J.-Y. Tourneret, and S. Bidon, "Knowledge-aided Bayesian detection in heterogeneous environments," IEEE Signal Processing Letters, vol. 14, no. 5, pp. 355-358, 2007. 
[37] A. De Maio, S. De Nicola, A. Farina, and S. Iommelli, "Adaptive detection of a signal with angle uncertainty," IET Radar, Sonar and Navigation, vol. 4, no. 4, pp. 537-547, 2010.

[38] A. De Maio and E. Conte, "Adaptive detection in gaussian interference with unknown covariance after reduction by invariance," IEEE Transactions on Signal Processing, vol. 58, no. 6, pp. 2925-2934, 2010.

[39] U. Nickel, "Design of generalised 2D adaptive sidelobe blanking detectors using the detection margin," Signal Processing, vol. 90, no. 5, pp. 1357-1372, 2010.

[40] W. R. Blanding, W. Koch, and U. Nickel, "Adaptive phasedarray tracking in ECM using negative information," IEEE Transactions on Aerospace and Electronic Systems, vol. 45, no. 1, pp. 152-166, 2009.

[41] M. Feldmann and U. Nickel, "Target parameter estimation and tracking with adaptive beamforming," in Proceedings of the International Radar Symposium (IRS '11), pp. 585-590, Leipzig, Germany, September 2011. 

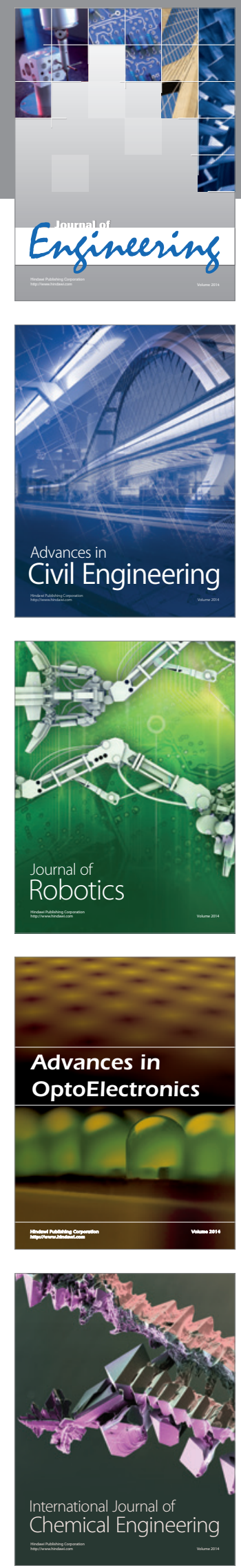

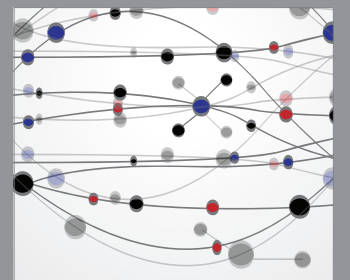

The Scientific World Journal
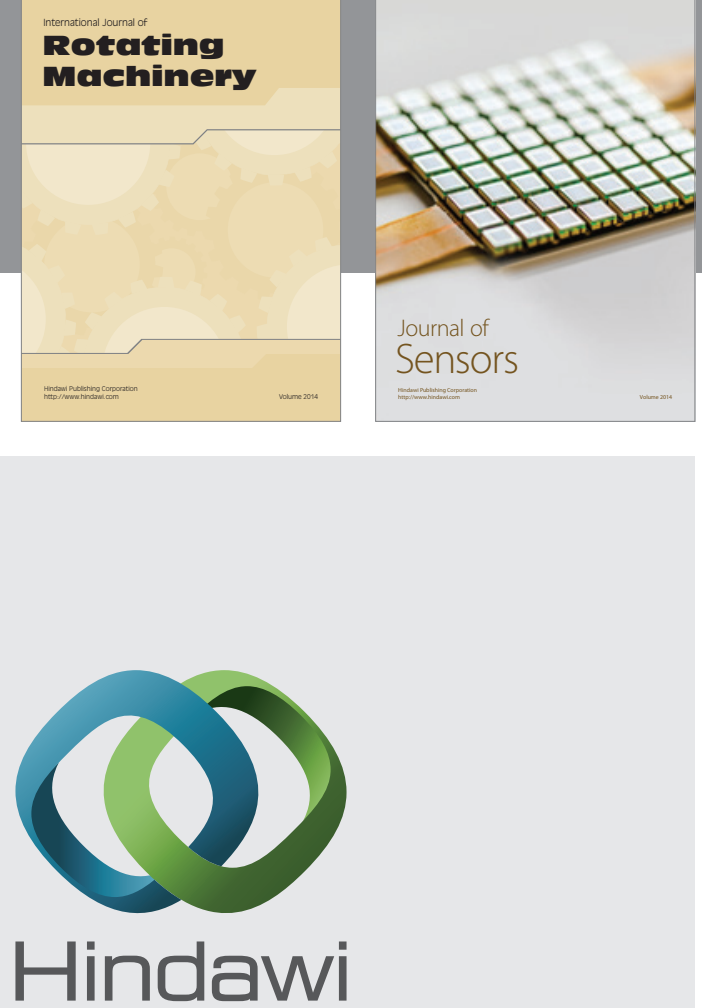

Submit your manuscripts at http://www.hindawi.com
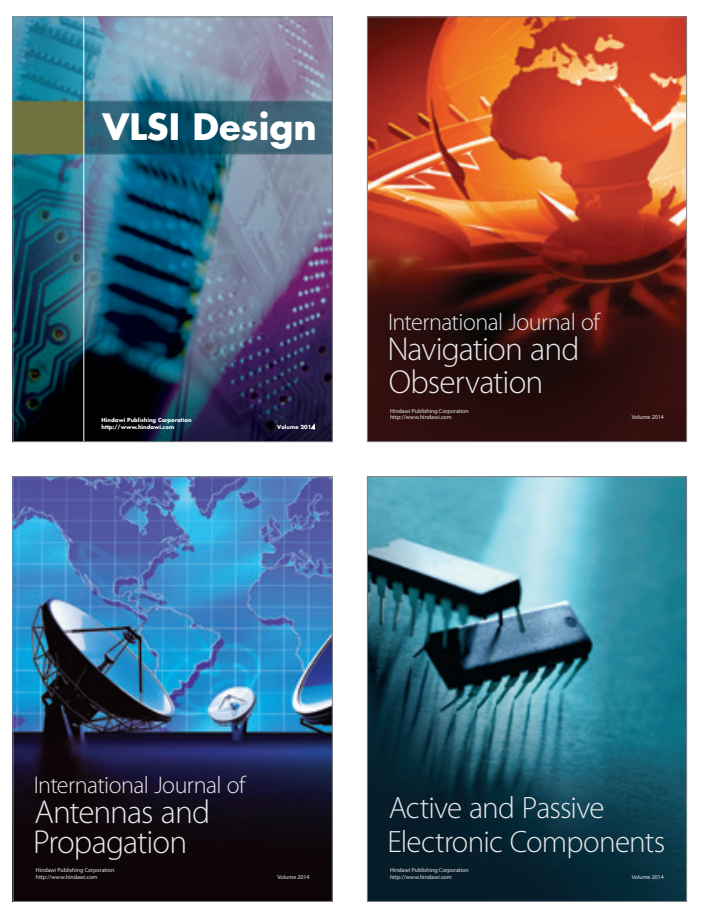
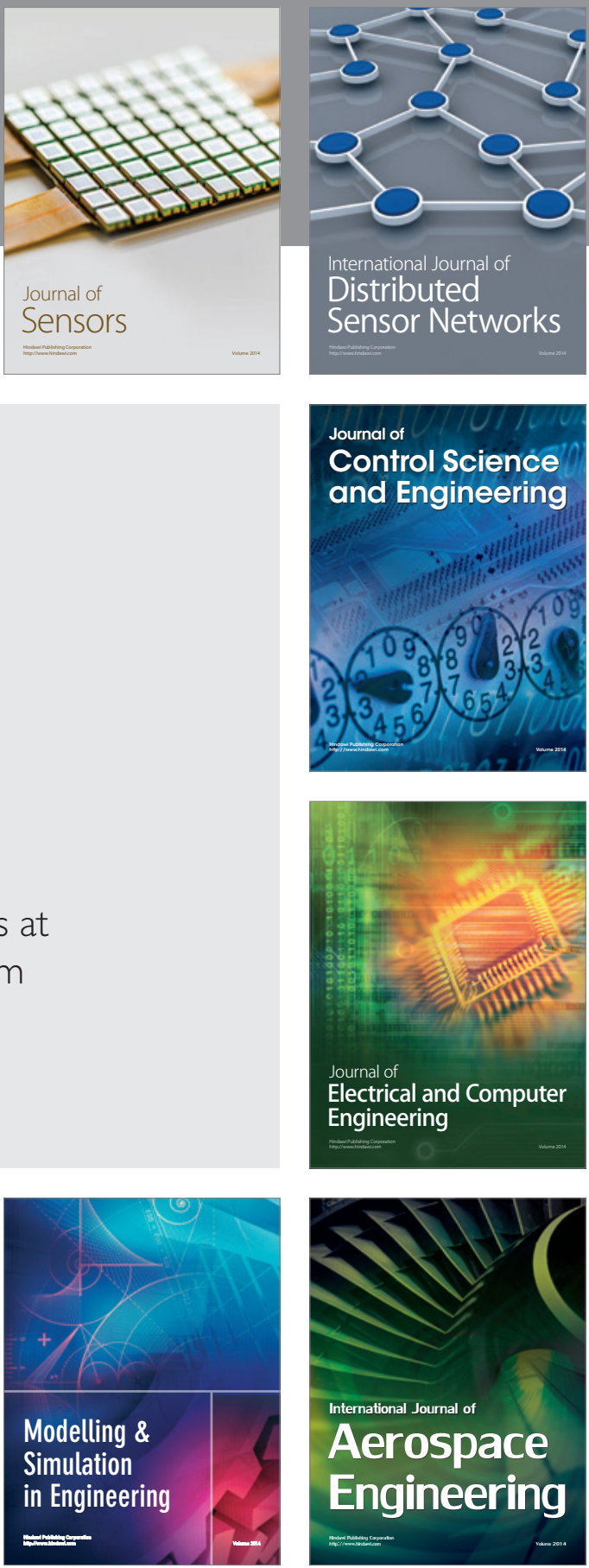

Journal of

Control Science

and Engineering
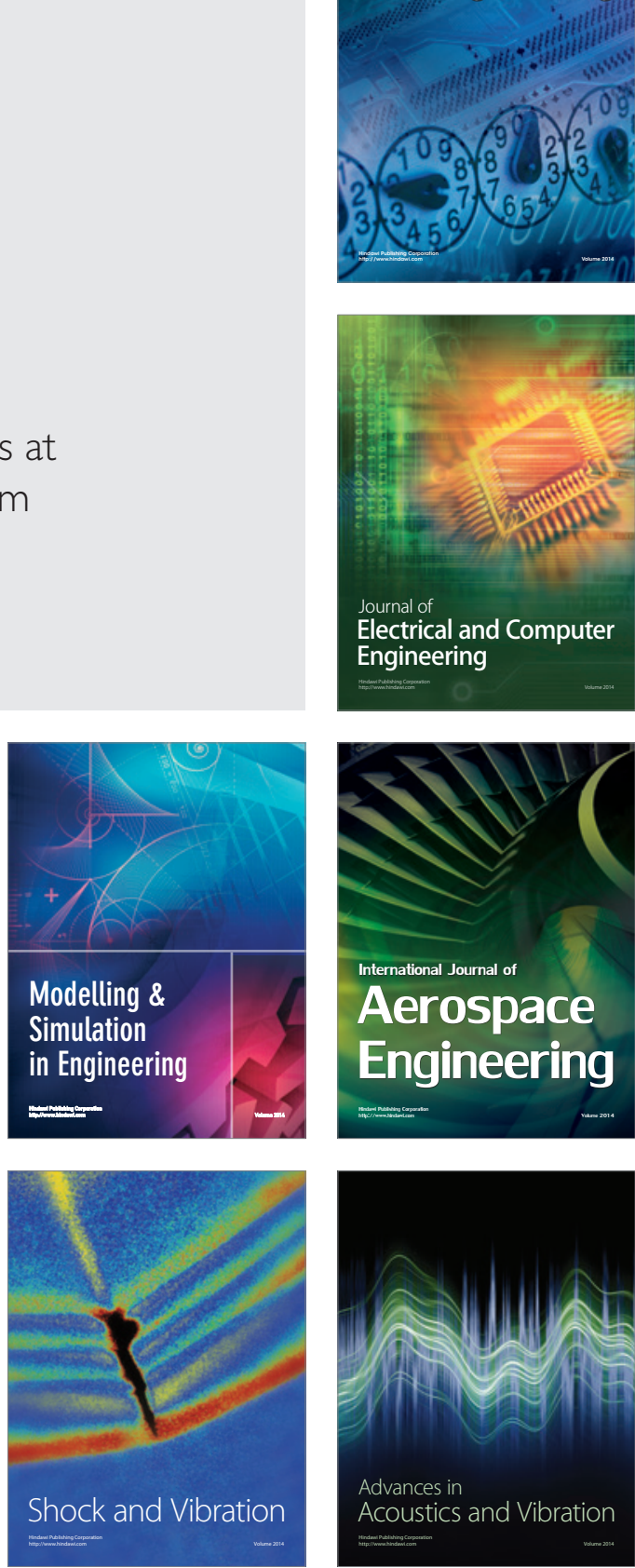